This item was submitted to Loughborough's Research Repository by the author.

Items in Figshare are protected by copyright, with all rights reserved, unless otherwise indicated.

\title{
Predicting real-time traffic conflicts using deep learning
}

PLEASE CITE THE PUBLISHED VERSION

https://doi.org/10.1016/j.aap.2019.105429

PUBLISHER

Elsevier

VERSION

AM (Accepted Manuscript)

PUBLISHER STATEMENT

This paper was accepted for publication in the journal Accident Analysis and Prevention and the definitive published version is available at https://doi.org/10.1016/j.aap.2019.105429.

\section{LICENCE}

CC BY-NC-ND 4.0

\section{REPOSITORY RECORD}

Formosa, Nicolette, Mohammed Quddus, Stephen Ison, Mohamed Abdel-Aty, and Jinghui Yuan. 2020. "Predicting Real-time Traffic Conflicts Using Deep Learning". figshare. https://hdl.handle.net/2134/11877687.v1. 
1 Predicting real-time traffic conflicts using deep learning

3 Nicolette Formosa, Corresponding author

4 School of Architecture, Building and Civil Engineering,

5 Loughborough University

6 Loughborough LE11 3TU United Kingdom

$7 \quad$ E-mail: N.Formosa@lboro.ac.uk

Mohammed Quddus

10 School of Architecture, Building and Civil Engineering,

11 Loughborough University

12 Loughborough LE11 3TU United Kingdom

13 Tel: +44(0)1509 228545

14 E-mail: M.A.Quddus@1boro.ac.uk

\section{Stephen Ison}

17 Department of Politics, People and Place,

18 Leicester Castle Business School,

19 The Gateway, Leicester, LE1 9BH, United Kingdom

20 Tel: +44(0)116257 7647

21 E-mail: Stephen.ison $@$ dmu.ac.uk

Mohamed Abdel-Aty

24 Department of Civil, Environmental and Construction Engineering,

25 University of Central Florida

26 Orlando, FL 32816, USA

27 Tel: +1(407)823-4535

28 E-mail: M.Aty@ucf.edu

\section{Jinghui Yuan}

31 Department of Civil, Environmental and Construction Engineering,

32 University of Central Florida

33 Orlando, FL 32816, USA

34 E-mail: jinghuiyuan@knights.ucf.edu 


\section{Abstract}

2 Recently, technologies for predicting traffic conflicts in real-time have been gaining momentum

3 due to their proactive nature of application and the growing implementation of ADAS

4 technology in intelligent vehicles. In ADAS, machine learning classifiers are utilised to predict

5 potential traffic conflicts by analysing data from in-vehicle sensors. In most cases, a condition is

6 classified as a traffic conflict when a safety surrogate (e.g. time-to-collision, TTC) crosses a pre-

7 defined threshold. This approach, however, largely ignores other factors that influence traffic

8 conflicts such as speed variance, traffic density, speed and weather conditions. Considering all

9 these factors in detecting traffic conflicts is rather complex as it requires an integration and

10 mining of heterodox data, the unavailability of traffic conflicts and conflict prediction models

11 capable of extracting meaningful and accurate information in a timely manner. In addition, the

12 model has to effectively handle large imbalanced data. To overcome these limitations, this paper

13 presents a centralised digital architecture and employs a Deep Learning methodology to predict

14 traffic conflicts. Highly disaggregated traffic data and in-vehicle sensors data from an

15 instrumented vehicle are collected from a section of the UK M1 motorway to build the

16 model. Traffic conflicts are identified by a Regional-Convolution Neural Network (R-CNN)

17 model which detects lane markings and tracks vehicles from images captured by a single front-

18 facing camera. This data is then integrated with traffic variables and calculated safety surrogate

19 measures (SSMs) via a centralised digital architecture to develop a series of Deep Neural

20 Network (DNN) models to predict these traffic conflicts. The results indicate that TTC, as

21 expected, varies by speed, weather and traffic density and the best DNN model provides an

22 accuracy of 94\% making it reliable to employ in ADAS technology as proactive safety

23 management strategies. Furthermore, by exchanging this traffic conflict awareness data,

24 connected vehicles (CVs) can mitigate the risk of traffic collisions.

25

26 Keywords: Safety Surrogate Measures, traffic conflicts, data integration architecture, Regional-

27 Convolution Neural Network (R-CNN), Deep Neural Network (DNN) 


\section{Introduction}

2 Recent breakthroughs in technology, digital infrastructure, machine learning and big data

3 computing will transform the way we will plan, undertake, interact, make decisions and use our

4 built environment, vehicles and transport infrastructure for the movement of people and goods.

5 Intelligent vehicles, enabled by connectivity and automation, cloud computing, artificial

6 intelligence and Internet of Things (IoT), will allow unprecedented capability to collect,

7 exchange and analyse large volumes of heterodox data for optimal decision making in real-time

8 and will therefore increasingly revolutionise our economy and society over the coming decades.

9 For instance, ADAS (advanced driver assistance system) technologies have the

10 potential to transform the automotive sector (estimated revenue of $\$ 89.3$ billion in 2025

11 according to Tractica, 2016) as they protect occupants, drivers and reduce collisions through

12 monitoring, warning, braking, and steering tasks. Since driver error contributes to over $90 \%$ of

13 traffic collisions (NHTSA, 2015), the underpinning principle of ADAS is to support the driver in

14 driving safely including reducing human involvement from the loop of driving as necessary.

15 Fuelled largely by regulatory and consumer interest in safety applications (for instance, both the

16 European Union and the United States are mandating that all vehicles be equipped with

17 autonomous emergency-braking systems and forward-collision warning systems by 2020), a

18 recent McKinsey survey suggests that consumers are becoming even more interested in ADAS

19 applications that promote comfort and economy, such as those that assist with parking or

20 monitoring blind spots (Mohr et al., 2013). ADAS utilises different vehicle-based safety

21 surrogate measures (SSMs) in order to warn drivers of any potential conflict. This is largely

22 based on a pre-specified threshold of a single metric - time-to-collision (TTC) or its modified

23 version rather than other important SSMs such as speed variance, yaw rate, lateral and 
1 longitudinal deceleration/acceleration rates, time gap and distance-to-collision along with their

2 variant by traffic density, speed and weather.

In addition, the automotive industry is currently designing and manufacturing

4 Autonomous Vehicles (AVs). However, at present, the safety aspect of these automated driving

5 has primarily been tackled with data and information gathered from in-vehicle sensors and

6 equipment. Given the fact that a sensor failure is inevitable even with redundant measurements

7 in a chain of operations, a holistic approach is required to guarantee a collision-free path

8 planning of automated driving (Katrakazas et al., 2017). For example, a conflict detection

9 technique may provide a critical hint of any collision-risk arising from the contextual and

10 circumstantial factors such as collision hot-spots identified through historical collision data,

11 unsafe traffic dynamics in real-time, infrastructure deficiencies, complex road layout, and other

12 imminent threats from surrounding objects. A real-time traffic conflict detection technique will

13 also be very important and useful for forming effective platoons in connected vehicles (CVs).

14 It is, therefore, fundamental to develop a real-time conflict detection method suitable for

15 ADAS, CVs and AVs. The overarching complexity in building such a model lies in the

16 management, integration and use of off-line and real-time data which are large and heterodox in

17 many ways such as uncertainty, sampling frequency, completeness, structure, consistency and

18 accuracy. In addition, an appropriate modelling technique, which can handle this large and

19 complex data, is required. Earlier modelling techniques have focused on nonparametric methods

20 and machine learning models, such as a regression tree model (Hossain and Muromachi, 2013),

21 support vector machine (Yu and Abdel-Aty, 2013) and genetic programming (Xu et al., 2013a).

22 Nonetheless, these techniques cannot perform the analysis required for big data with complex

23 characteristics and have not addressed the issues emerging when the data is highly imbalanced. 
1 Therefore, the results emerging from such techniques are inclined to be profoundly influenced by

2 the large classes (e.g. 'safe' traffic dynamics) while neglecting the smaller ones (e.g. traffic

3 conflicts). This is because they aim to minimise the overall error rate rather than paying specific

4 attention to the smaller classes. On the other hand, Deep Learning (DL) in particular Deep

5 Neural Network (DNN) models with a Mean Squared False Error loss function have the ability

6 to capture classification errors from both majority and minority classes equally (Wang et al.,

7 2016a). They are also able to elicit complex and non-linear patterns to classify and predict while

8 catering for big data (Najafabadi et al., 2015). Therefore, DL methodology would be an ideal

9 candidate in addressing the complexities discussed earlier.

10 The purpose of this paper is two-fold: (1) to design a data integration architecture for

11 heterodox data and (2) to develop a real-time traffic conflict detection method using DL

12 methodology. A section of the M1 motorway in the UK was used as a case study from which

13 data was collected using an instrumented vehicle. Due to the large complex datasets collected

14 from multiple sensors, a data integration architecture was developed to extract meaningful and

15 accurate information in a timely manner. From the video data, the safe traffic dynamics and

16 traffic conflicts were identified and together with the other collected, and estimated factors

17 (SSMs and traffic variables), the DNN model was developed. Parts of the data were utilised to

18 validate the performance of models using K-fold cross-validation and to determine the

19 performance and ability of the model in predicting link-level traffic conflicts.

20 2. Literature Review

21 Timely detection of traffic conflicts is fundamental to proactively mitigate the risk of collision

22 through ADAS, CVs and AVs. This has been very challenging as multiple factors act

23 concurrently and the methods employed to detect conflicts have some severe weaknesses. This 
1 review is therefore structured into two main parts: factors affecting conflicts and methods to

2 detect them.

\section{$3 \quad 2.1 \quad$ Factors affecting real-time conflicts}

4 SSMs are used as an indirect measure of traffic safety as they can be effectively utilized to

5 proactively identify safe traffic dynamics and traffic conflicts. By understanding how different

6 measures interact with each other in developing a traffic conflict, evasive manoeuvres are

7 recommended by proactive safety systems such as ADAS. In fact, a traffic conflict is identified

8 when the value of a SSM exceeds a critical threshold (Zaki et al., 2013).

9 There are different property based SSMs adopted to estimate the likelihood that an unsafe

10 condition arises. In the case of estimating risk behaviours, the time-based SSMs have been

11 adopted since they unify both spatial proximity and speed difference (Laureshyn et al., 2010).

12 However, the time-based SSMs are not always sensitive for spatial proximity, when the

13 maximum braking capacity is applied. In this case, distance-based SSMs have found to be

14 appropriate since the risk of a collision is relatively high if the required distance for executing an

15 evasive action is less than the available distance. On the other hand, when an evasive manoeuvre

16 in a traffic conflict is detected, such as braking, SSMs based on deceleration are important to

17 assess the kinematic characteristics involving risk avoidance. As a result, each property-based

18 conflict indicators represent partial images of the true severity of critical traffic events. Typical

19 SSMs based on three properties as identified in the literature are presented in Table 1. These

20 SSMs are primarily related to car-following and lane-changing related traffic conflicts. For each

21 of the SSMs, the definition, critical value and additional comments are presented.

\section{Table 1}

23 SSM factors 


\begin{tabular}{|c|c|c|c|}
\hline$\overline{\text { SSM }}$ & Definition & Critical Value & Comments \\
\hline $\begin{array}{l}\text { Post- } \\
\text { Encroachment } \\
\text { Time (PET) } \\
\text { Time-based }\end{array}$ & $\begin{array}{l}\text { The time taken for the rear } \\
\text { bumper of the preceding } \\
\text { vehicle to leave the zone of a } \\
\text { common spatial zone till the } \\
\text { front bumper of the second } \\
\text { vehicle enters the same zone } \\
\text { (Songchitruksa and Tarko, } \\
\text { 2006). }\end{array}$ & $\begin{array}{l}\text { A PET value less } \\
\text { than } 1 \text { or } 1.5 \\
\text { seconds is } \\
\text { considered as } \\
\text { critical (Archer, } \\
\text { 2005). }\end{array}$ & $\begin{array}{l}\text { This measure while reflecting the } \\
\text { drivers' behavior, does not consider } \\
\text { the impact of the collision. } \\
\text { PET } \approx \frac{\mathrm{R}}{\mathrm{V}_{\mathrm{FV}}}\end{array}$ \\
\hline $\begin{array}{l}\text { Time To } \\
\text { Collision (TTC) } \\
\text { Time-based }\end{array}$ & $\begin{array}{l}\text { The remaining time before an } \\
\text { impact takes place between } \\
\text { two road users unless an event } \\
\text { to change their present } \\
\text { physical parameters occurs. }\end{array}$ & $\begin{array}{l}\text { The critical value } \\
\text { for TTC is given as } \\
1.5 \text { seconds. }\end{array}$ & $\begin{array}{l}\text { An assumption is that the } \\
\text { consecutive vehicles have to sustain } \\
\text { constant speeds. } \\
\text { TTC }=\frac{\mathrm{R}-\mathrm{L}}{\mathrm{V}_{\mathrm{F}}-\mathrm{V}_{\mathrm{L}}} \quad \forall \mathrm{V}_{\mathrm{FV}}>\mathrm{V}_{\mathrm{PV}}\end{array}$ \\
\hline $\begin{array}{l}\text { Modified TTC } \\
\text { (MTTC) } \\
\text { Time-based }\end{array}$ & $\begin{array}{l}\text { This SSM proposed by } \\
\text { (Ozbay et al., 2008) follows } \\
\text { the TTC indicator but } \\
\text { considers combinations of } \\
\text { relative speeds and } \\
\text { accelerations in the various } \\
\text { car following scenarios. } \\
\end{array}$ & $\begin{array}{l}\text { A } 4 \text { second } \\
\text { threshold value for } \\
\text { the MTTC measure } \\
\text { was proposed in } \\
\text { (Ozbay et al., } \\
\text { 2008). }\end{array}$ & $\begin{array}{l}\text { The MTTC value by itself does not } \\
\text { provide enough indication about the } \\
\text { seriousness of the collision. } \\
\text { MTTC } \\
=\frac{-\Delta \mathrm{V} \pm \sqrt{(}\left(\Delta \mathrm{V}^{2}+2 \Delta \mathrm{a} \Delta \mathrm{X}\right)}{\Delta \mathrm{a}}\end{array}$ \\
\hline $\begin{array}{l}\text { Proportion of } \\
\text { Stopping } \\
\text { Distance (PSD) } \\
\text { Distance-based }\end{array}$ & $\begin{array}{l}\text { The ratio between the } \\
\text { remaining distance (RD) } \\
\text { based on the assumption that } \\
\text { the maximum deceleration } \\
\text { rate is used to the potential } \\
\text { point of collision and the } \\
\text { Minimum acceptable } \\
\text { Stopping Distance (MSD). }\end{array}$ & $\begin{array}{l}\text { A PSD value less } \\
\text { than } 1 \text {, is regarded } \\
\text { as 'unsafe' and } \\
\text { collisions cannot be } \\
\text { avoided if the } \\
\text { MADR is } \\
\text { considered }\end{array}$ & $\begin{array}{l}\text { PSD is regarded as a good surrogate } \\
\text { indicator and has been used for } \\
\text { safety evaluation by (Guido and } \\
\text { Saccomanno, 2010). } \\
\quad \text { PSD }=\frac{\mathrm{RD}}{\mathrm{MSD}} \quad \mathrm{MSD}=\frac{\mathrm{V}_{\mathrm{FV}}^{2}}{2 \mathrm{a}_{\mathrm{FV}}^{\min }}\end{array}$ \\
\hline $\begin{array}{l}\text { Deceleration } \\
\text { Rate to Avoid a } \\
\text { Crash (DRAC) } \\
\text { Deceleration- } \\
\text { based }\end{array}$ & $\begin{array}{l}\text { The rate at which the } \\
\text { following vehicle must } \\
\text { decelerate to come to a timely } \\
\text { stop to prevent a collision } \\
\text { with other conflicting vehicles } \\
\text { or to obtain the same speed of } \\
\text { preceding vehicle to avoid a } \\
\text { rear-end collision (Xiaobo Qu } \\
\text { et al., 2014) }\end{array}$ & $\begin{array}{l}\text { A DRAC value of } \\
\text { more than } 3.35 \mathrm{~m} / \mathrm{s}^{2} \\
\text { is considered as } \\
\text { being in a traffic } \\
\text { conflict (Archer, } \\
\text { 2005). }\end{array}$ & $\begin{array}{l}\text { DRAC gives a better measure to } \\
\text { represent both frequency and } \\
\text { severity of high-risk vehicle } \\
\text { interactions. Many researchers have } \\
\text { acknowledged the relevance of } \\
\text { DRAC as a safety-performance } \\
\text { measure, e.g.(Archer, 2005; Guido } \\
\text { and Saccomanno, 2010). } \\
\text { DRAC } \\
=\left\{\begin{aligned} \frac{\left(\mathrm{V}_{\mathrm{FV}}-\mathrm{V}_{\mathrm{PV}}\right)^{2}}{\Delta \mathrm{X}}, & \text { if } \mathrm{V}_{\mathrm{FV}}>\mathrm{V}_{\mathrm{PV}} \\
0, & \text { otherwise }\end{aligned}\right.\end{array}$ \\
\hline $\begin{array}{l}\text { Criticality Index } \\
\text { Function (CIF) } \\
\text { Deceleration- } \\
\text { based }\end{array}$ & $\begin{array}{l}\text { This aim of this index is to } \\
\text { consider the degree of danger } \\
\text { and the probability of an } \\
\text { imminent conflict even if a } \\
\text { collision does not essentially } \\
\text { occur (Chan, 2006). }\end{array}$ & Same as TTC & $\begin{array}{l}\text { This index can be used as a surrogate } \\
\text { safety measure to evaluate the } \\
\text { probability and the severity of a } \\
\text { collision. } \\
\qquad \mathrm{CIF}=\frac{\mathrm{V}_{\mathrm{FV}}^{2}}{\mathrm{TTC}}\end{array}$ \\
\hline
\end{tabular}


1 In Table $1, \mathrm{R}$ is the range of the two vehicles, $\mathrm{L}$ is the length of preceding vehicle, $\Delta \mathrm{X}$ is $(\mathrm{R}-\mathrm{L})$,

$2 \mathrm{~V}_{\mathrm{FV}}$ and $\mathrm{V}_{\mathrm{PV}}$ denote the speed of the following and preceding vehicle respectively, MADR is the 3 maximum available deceleration rate and $\mathrm{aFv}^{\mathrm{min}}$ is typically set as $3.92 \mathrm{~m} / \mathrm{s}^{2}$.

4 Nevertheless, traffic variables also play an important role in predicting crash occurrences, 5 and the quantification of their impact has been utilised to formulate countermeasures in

6 improving traffic safety (Wang et al., 2015). This is because unsafe road occurrences take place

7 when traffic moves, and it is, therefore, natural to explore traffic characteristics to understand

8 their impact on dangerous conditions. In fact, many studies have been carried out to analyse the

9 connection between a potential crash probability and various traffic parameters (Xu et al.,

10 2013b). A few patterns have been observed in the literature about how each variable affects

11 unsafe conditions, despite that mixed evidence is presented. These traffic variables are inter-

12 related and are usually classified as traffic speed, traffic flow, density and occupancy (Wang et

13 al., 2013). Moreover, recent research indicates that SSMs are heavily influenced by traffic

14 variables. For instance, Papazikou et al. state that critical TTC values are different at different

15 operating speeds of the roadway (Papazikou et al., 2017).

16 Therefore, by embedding these traffic variables in conjunction with the SSMs as factors

17 in proactive safety systems to predict traffic conflicts may be beneficial. This adds more

18 information about the network and the environment through which the ego-vehicle is driving.

19 The inherent challenge in developing such a safety automotive system is that intelligent vehicles

20 are equipped with a growing number of complex components which need to run simultaneously

21 while also being time-critical because of the safety requirements (Zheng et al., 2016). These

22 components include the sensors used such as radar, camera and GPS to estimate the SSMs and to

23 collect real-time traffic variables. This data also needs to be processed using computational units 
1 such as processors and GPUs. These sensors and computational units are then connected through

2 various buses while spanning different languages, interfaces and hardware and processing a high

3 volume of data to perceive the environment accurately and efficiently. Therefore, such systems

4 need to adopt a valid and reliable data integration architecture system in order to process large

5 and heterodox data in real-time.

\section{$6 \quad 2.2 \quad$ Modelling techniques for real-time conflict detection}

7 A fundamental challenge when it comes to predicting rare events in the real world is the unequal

8 distribution between its classes creating a highly imbalanced dataset (He and Garcia, 2009). In

9 this study, traffic conflicts are also observed less frequently than safe traffic dynamics. This can

10 compromise the performance of some machine learning predictors since they are based on the

11 assumption of balanced class distributions. To overcome this issue, studies identified in the

12 literature have made use of case-control designs when developing prediction models (e.g. Abdel-

13 Aty et al., 2005). However, since some of the data has been omitted, it may influence the

14 calibration of the model resulting in an inaccurate output. To obtain a more realistic and reliable

15 prediction from the machine learning predictor, which genuinely reflects the underlying

16 classification a full dataset should, therefore, be adopted (Yang et al., 2018).

17 Existing studies have mainly used shallow prediction models, which cannot be applied to

18 the real-world complex highly imbalanced dataset. On the other hand, in work by Sameen and

19 Pradhan (2017), a DL approach is adopted, and their research reveals the capability of this

20 methodology to overcome the data imbalance problem. Yang et al. (2018) also address this

21 imbalance problem by using a DL approach. Again, their results achieve a powerful

22 identification ability in real-time prediction. Hence since the data used in this study is complex,

23 large, heterodox and highly imbalanced, a DL methodology is adopted. Additionally, this study 
1 takes advantage of its superior performance and the powerful ability to tackle high-dimensional

2 data.

3 In summary, it has been revealed that there is a considerable amount of work undertaken

4 in the identification of the factors which contribute to traffic conflicts. However, these studies at

5 best provide mixed conclusions about their efficiency and applicability. This highlights the need

6 for the use of more than one factor so as to get a clear understanding of the conflict mechanism

7 (Laureshyn et al., 2010). Therefore, by adding both the traffic variables with the SSMs as factors

8 to develop a model to predict traffic conflicts this increases the system's reliability and efficiency

9 (Nadimi et al., 2016). However, this is not a simple task as it requires a reliable centralised data

10 architecture system to allow for the data from sensors to be processed, synchronised and

11 embedded as factors in the model. Nevertheless, this data is highly complex and imbalanced, and

12 a DL methodology has to be adopted to cater for this data.

\section{3. Development of a Data Integration Architecture and Data Collection}

14 Real-time vehicle kinematics and traffic conditions data have been collected using an

15 instrumented vehicle. However, a large amount of data cannot be processed using conventional

16 systems because of many forms of data, their complexity and their varying sampling frequencies.

17 Hence, to examine the information and patterns within the data, a system architecture is needed

18 for data collection, transmission and storage. In order to unify and process all the information on

19 a central computational unit, a centralised data integration architecture was developed to satisfy

20 the requirements of various sensor environments and provide a run-time environment. The

21 advantage of this architecture is that the combination and arrangement of the system allow all the

22 functions to share information more conveniently. Nevertheless, the centralised architecture

23 requires high computation capability. The architecture transmits two kinds of information: 
1 continuous information of the vehicles localisation and velocity, and information about the

2 surrounding dynamic environment. It is developed as shown in Figure 1:

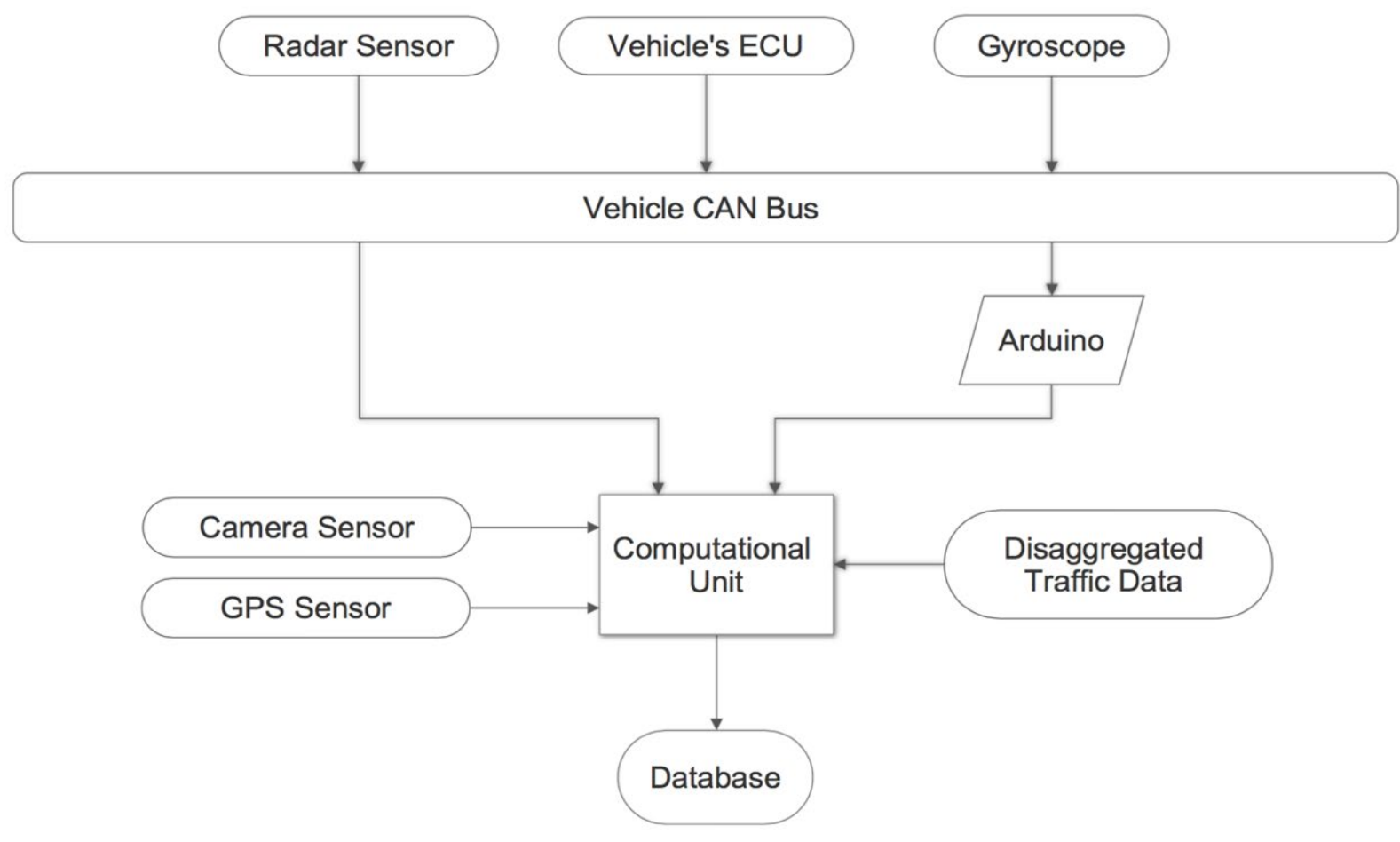

$4 \quad$ Fig. 1. Centralised data integration architecture

5 In this study, the vehicle's speed and the yaw rate are collected by the Electronic Control Unit

6 (ECU), and the gyroscope respectively by scanning the Controller Area Network (CAN) bus at a

7 rate of $15 \mathrm{~Hz}$. To increase the accuracy and quality of the data, these messages are then linked to

8 the radar sensor messages. The vehicle speed computed from the radar sensor alone is not

9 sufficient. This is because, if the ego-vehicle is travelling at the same speed as one of the

10 detected vehicles the latter will appear static. However, by noting the speed of the ego-vehicle,

11 the actual speed of the detected vehicle can be computed. These combined messages are then

12 read via an Arduino microcontroller and transferred via a USB to a computational unit where all

13 the other sensors (Radar, Camera and GPS) and disaggregated traffic data are connected to the

14 same computational unit. 
The instrumented vehicle is installed with a radar sensor (ARS 308-21) and a

2 Grasshopper3 camera (GS3-U3-41C6C-C). The localisation sensor installed is a U-Blox EVK-

$3 \quad$ M8 GNSS system with DR which enables simple evaluation of the high-performance positioning

4 technology. The disaggregate traffic flow data is obtained from Motorway Incident Detection

5 and Automatic Signalling (MIDAS). Each loop detector records traffic information such as

6 vehicle speed, occupancy, flow and headway. The information is defined per minute by lane and

7 collected from the 88 loop detectors found in the study area with a spacing of nominally $400 \mathrm{~m}$.

8 The data is obtained from the loop detector with the lowest Euclidean distance between the ego-

9 vehicle and the loop detector. A summary of the measures from sensors and loop detectors are

10 presented in Table 2.

11 Some of the issues associated with data collection relate to: missing data,

12 communication problems and spurious data due to hardware or software problems. Another issue

13 for loop detector data is the 'no-flow' data when no vehicles appeared within the current minute

14 (O’Reilly et al., 2004). Some errors can be observed from unrealistic values for example:

15 (i) speed values $<0 \mathrm{~km} / \mathrm{hr}$,

16 (ii) occupancy $<0 \%$ or $>100 \%$,

17 (iii) flow $=0 \mathrm{veh} / \mathrm{min}$ and speed $>0 \mathrm{~km} / \mathrm{hr}$,

18 (iv) flow $=0 \mathrm{veh} / \mathrm{min}$, speed $=0 \mathrm{~km} / \mathrm{hr}$ and occupancy $>0 \%$,

19 (v) flow $>0 \mathrm{veh} / \mathrm{min}$ and speed $=0 \mathrm{~km} / \mathrm{hr}$,

20 (vi) flow $>0 \mathrm{veh} / \mathrm{min}$, speed $>0 \mathrm{~km} / \mathrm{hr}$ and occupancy $=0 \%$.

All the observations from these sensors were gathered in their respective program, are

22 then extracted from the sensing system and stored in a database. The data from each sensor is 
1 synchronised creating a centralised integrated architecture in which all data, measurement

2 readings of multiple variables is gathered in one place rather than on multiple computation units.

3 Table 2

$4 \quad$ List of Measures

\begin{tabular}{|c|c|c|c|}
\hline & $\begin{array}{l}\text { Variables based on } \\
\text { Source }\end{array}$ & Description and Use & Units \\
\hline \multirow{15}{*}{ 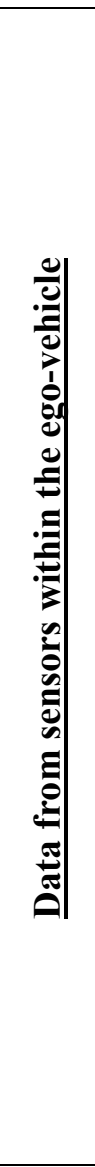 } & \multicolumn{3}{|c|}{ "GPS (Frequency of 1Hz) } \\
\hline & Position - Ego $(\mathrm{X}, \mathrm{Y})$ & $\begin{array}{l}\text { Longitude and Latitude which specify the precise } \\
\text { location of the ego vehicle on the surface of the Earth. }\end{array}$ & $\% / \mathrm{min}$ \\
\hline & Speed - Ego & The rate of change of position with time. & $\mathrm{m} / \mathrm{s}$ \\
\hline & \multicolumn{3}{|c|}{$\begin{array}{l}\text { Vehicle's ECU and Gyroscope messages sent through the CAN Bus and read using an } \\
\text { Arduino (Frequency of } 15 \mathrm{~Hz} \text { ) }\end{array}$} \\
\hline & Velocity - Ego & The rate of change of position with time. & $\mathrm{m} / \mathrm{s}$ \\
\hline & Acceleration - Ego & The rate of change of velocity with time. & $\mathrm{m} / \mathrm{s}^{2}$ \\
\hline & Yaw Rate - Ego & The rate of change of angular velocity. & $\% / \mathrm{s}$ \\
\hline & \multicolumn{3}{|c|}{ Radar (Frequency of $15 \mathrm{~Hz}$ with a range of $200 \mathrm{~m}$ and azimuth angle of $56^{\circ}$ ) } \\
\hline & $\begin{array}{l}\text { Longitudinal } \\
\text { Displacement }\end{array}$ & $\begin{array}{l}\text { Range in the } \mathrm{x} \text {-direction between the target and the } \\
\text { ego-vehicle. }\end{array}$ & $\mathrm{m}$ \\
\hline & Lateral Displacement & $\begin{array}{l}\text { Range in the y-direction. Determines lane } \\
\text { classification. }\end{array}$ & $\mathrm{m}$ \\
\hline & $\begin{array}{l}\text { Relative Longitudinal } \\
\text { Speed }\end{array}$ & $\begin{array}{l}\text { Relative speed between the ego and the target vehicle; } \\
\text { from which the speed of the target vehicle can be } \\
\text { calculated. }\end{array}$ & $\mathrm{m} / \mathrm{s}$ \\
\hline & $\begin{array}{l}\text { Relative Lateral } \\
\text { Velocity }\end{array}$ & $\begin{array}{l}\text { The component of the velocity of the target vehicle } \\
\text { which is resolved in the y-direction. }\end{array}$ & $\mathrm{m} / \mathrm{s}$ \\
\hline & $\begin{array}{l}\text { Relative Lateral } \\
\text { Acceleration }\end{array}$ & $\begin{array}{l}\text { Relative acceleration between the ego and the target } \\
\text { vehicle; from which the acceleration of the target } \\
\text { vehicle can be calculated. }\end{array}$ & $\mathrm{m} / \mathrm{s}^{2}$ \\
\hline & \multicolumn{3}{|c|}{ Camera (Frequency of $15 \mathrm{~Hz})$} \\
\hline & Video data & $\begin{array}{l}\text { Record surrounding road traffic environment at an } \\
\text { image resolution of } 2048 \times 2048\end{array}$ & fps \\
\hline \multirow{5}{*}{ 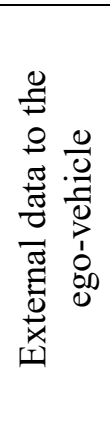 } & \multicolumn{3}{|c|}{ Loop Detectors (per lane) (Frequency of 0.0167Hz i.e. 1-min interval) } \\
\hline & Speed & The average speed for each lane & $\mathrm{km} / \mathrm{hr}$ \\
\hline & Flow & $\begin{array}{l}\text { The total number of vehicles passing through each } \\
\text { lane }\end{array}$ & vehicles/min \\
\hline & Occupancy & $\begin{array}{l}\text { The percentage of time the monitoring site space was } \\
\text { occupied by a vehicle for each lane }\end{array}$ & $0-100 \%$ \\
\hline & Headway & $\begin{array}{l}\text { The difference between the time when the front of } \\
\text { a vehicle arrives at a point and the time the front } \\
\text { of the next vehicle arrives at the same point }\end{array}$ & $\mathrm{s}$ \\
\hline
\end{tabular}


The data used in this study was collected from a section of the UK M1 motorway

2 between junctions 17 and 23 . The total length of this corridor is about $55 \mathrm{~km}$ with $3-4$ lanes on

3 each side and ten slip roads. A total of 15 trips were conducted between April 2017 and

4 December 2018 at different times between $10 \mathrm{am}$ and $7 \mathrm{pm}$ from multiple drivers. This is because

5 it is known that drivers behave differently under diverse traffic and environmental conditions. As

6 a result, data was also collected in various traffic conditions such as highly congested and free

7 flow conditions as well as in different weather conditions to cover most of the events that could

8 occur in the real-world. The total duration of these trips adds up to approximately 19 hours of

9 data with 1,200,000 observations at $15 \mathrm{~Hz}$. Since the instrumented vehicle has only a forward-

10 facing radar and camera, we can only study traffic conflicts associated with car-following and

11 lane-changing.

12 4. Methodology

13 Developing a conflict detection model is challenging as data on traffic conflicts and their

14 influencing factors are required. However, traffic conflicts data are not available and therefore, a

15 method is developed to identify traffic conflicts using data from an instrumented vehicle (see

16 Figure 1). The conflict identification process from the collected data consists of training an R-

17 CNN network to identify unsafe conditions by further detecting vehicle and lane geometry data

18 acquired through image processing techniques from a single front-facing camera. This data is

19 later integrated with the data from other sensors and fed into a DNN model to be trained in order

20 to predict traffic conflicts on the road. The methodology of this study consists of two main

21 components: (1) conflict identification and (2) conflict detection. A functional block diagram of

22 the methodology undertaken for conflict identification and classifier development is presented in

23 Figure 2. 


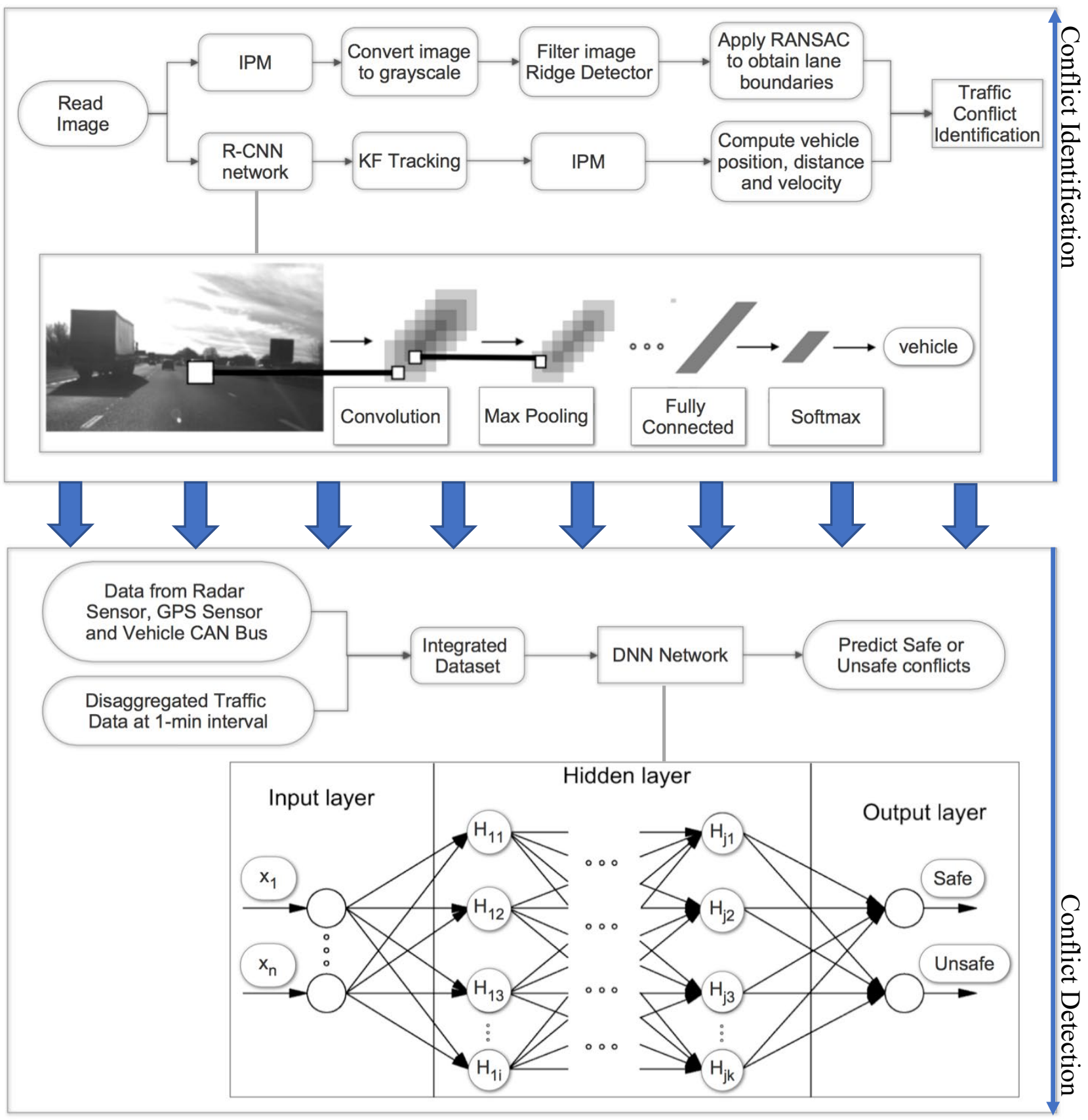

Fig. 2. The functional block diagram of the methodology undertaken

\section{$4 \quad 4.1 \quad$ Conflict event identification}

5 A new method is developed to identify traffic conflicts based on the data collected from our

6 instrumented vehicle. In order to identify traffic conflicts on the road, preceding vehicles were

7 detected, tracked and classified accordingly. Initially the video data is segregated into a large 
1 number of images. Ground truth labels on these images to train and test the model is necessary

2 for the model to be successful. However, obtaining high quality labelled data is costly and time-

3 consuming (Yamashita et al., 2018). A CNN learning classifier is trained to detect vehicles using

4 this ground truth data set, by combining bottom-up regional proposals with rich features (R-

5 CNN) (Zhao et al., 2018).

6 The building blocks of an R-CNN model includes convolution layers, pooling layers and

7 fully connected layers. A typical architecture is made up of repetition of two types of layers:

8 convolutional layers and pooling layers, followed by one or more fully connected layers. The

9 role of the convolutional layer is to carry out feature extraction by applying a kernel across the

10 input made from an array of numbers usually termed as tensor. Each element of the kernel is

11 multiplied to every input tensor and summed to obtain an output value organised in feature maps.

12 This procedure is repeated by applying multiple kernels to form a number of feature maps. These

13 feature maps represent different characteristics of the input tensors. Two key hyperparameters

14 that define the convolution operation are kernel size and the number of kernels. These are

15 typically chosen based on training dataset. An important note is that in order to fit the centre of

16 the kernel at the outermost element and to retain the same in-plane dimensions zero padding is

17 added to this layer. Moreover, the distance between two successive kernel positions (stride) is

18 often of value 1 . The result are then passed through a non-linearity activation function such as a

19 rectified linear unit (ReLU), identified as the half-wave rectifier $f(z)=\max (z, 0)$.

A pooling layer offers a process which reduces the in-plane dimensionality of the feature

21 maps in order to introduce a translation invariance to small shifts and distortions and decrease

22 the number of successive learnable parameters (Yamashita et al., 2018). In this particular layer,

23 there are no learnable parameters, whereas filter size, stride, and padding are hyperparameters in 
1 pooling operations, similar to convolution operations. Max pooling is the pooling operation

2 adopted in this research. It extracts patches from the input feature maps and uses only the

3 maximum value in each patch, while deleting the other values. A commonly used max pooling

4 layer is with a filter of size $2 \times 2$ with a stride of 2 . This decreases the sample size of the in-plane

5 dimension of feature maps by a factor of 2 . While this affects the height and width, the depth

6 dimension of feature maps is retained.

7 The output feature maps of the final convolution or pooling layer is usually flattened in

8 which every input is connected to every output by a weight. The fully connected layers are the

9 fundamental building blocks of the R-CNN model and, the composition of each layer creates a

10 highly non-linear system. Every layer is trained using stochastic gradient descent (SGD),

11 consisting in presenting the input vector with examples, estimating the outputs and the errors,

12 computing the average gradient for those examples, and adjusting the weights accordingly. The

13 process is iterated until the average of the objective function stops decreasing. It is described as

14 stochastic since each set of examples provided a noisy estimate of the average gradient over all

15 examples. This procedure usually finds a good set of weights, so the network is able to extract

16 features and learn the different sizes and shapes of the vehicles. Every fully connected layer is

17 also followed by a nonlinear function. The final layer outputs bounding boxes indicating the

18 regions occupied by a vehicle and the corresponding confidence.

19 A summary of the list of parameters (optimised during training) and hyperparameters (set

20 beforehand) in an R-CNN with the values used in this study is given in Table 3.

\section{Table 3}

22 List of parameters and hyperparameters in an R-CNN 


\begin{tabular}{|c|c|c|}
\hline $\mathrm{R}-\mathrm{CNN}$ & Parameters & Hyperparameters with values \\
\hline Convolution layer & Kernels & $\begin{array}{ll}\text { - } & \text { Kernel size }(3 \times 3) \\
\text { - } & \text { No. of kernels (random) } \\
\text { - } & \text { Stride }(1) \\
\text { - } & \text { Padding }(0) \\
\text { - } & \text { Activating function (ReLU) }\end{array}$ \\
\hline Pooling layer & & $\begin{array}{ll}\text { - } & \text { Filter Size }(2 \times 2) \\
\text { - } & \text { Stride }(2) \\
\text { - } & \text { Padding }(0)\end{array}$ \\
\hline Fully connected layer & Weights & $\begin{array}{ll}\text { - } & \text { No. of weights } \\
\text { - } & \text { Activation function (ReLU) }\end{array}$ \\
\hline Training process & & $\begin{array}{ll}\text { - } & \text { Model architecture (15 layers) } \\
\text { - } & \text { Optimizer } \\
\text { - } & \text { Learning Rate (1e-4) } \\
\text { - } & \text { Loss function (SGD) } \\
\text { - } & \text { Mini-batch size (128) } \\
\text { - } & \text { Epochs (60) } \\
\text { - } & \text { Regularisation } \\
\text { - } & \text { Weight initialisation (random) }\end{array}$ \\
\hline
\end{tabular}

Since the R-CNN model is not pixel accurate and the position and size of bounding box

3 changes, the results from this model can be noisy. To filter this noise, the position and

4 dimensions of bounding boxes are tracked with a Kalman Filter (KF) having a Constant Velocity

5 state transition model (Li and Jilkov, 2003). The KF also has the ability to predict how these

6 measurements will change in time by making use of a sequence of measurements and a

7 mathematical model. Hence, it also provides the prediction of the position and size of the

8 bounding boxes which increases the certainty within the results. This is because these can then

9 be compared with the actual visual in the next time step. The KF procedure is given in two stages

10 as follows:

11 Prediction Stage: 
$1 \quad \hat{\mathrm{x}}_{t \mid t-1}=\mathrm{A} \hat{\mathrm{x}}_{t-1}$

$2 \quad \mathrm{P}_{t \mid t-1}=\mathrm{AP}_{t} \mathrm{~A}^{\prime}+\mathrm{Q}$

3 Correction Stage:

$4 \quad \mathrm{~K}_{\mathrm{t}}=\mathrm{P}_{t \mid t-1} \mathrm{C}^{\prime}\left(\mathrm{CP}_{t \mid t-1} \mathrm{C}^{\prime}+\mathrm{R}\right)^{-1}$

$5 \quad \hat{\mathrm{x}}_{t}=\hat{\mathrm{x}}_{t \mid t-1}+\mathrm{K}_{t}\left(\mathrm{y}_{t}-\mathrm{C} \hat{\mathrm{x}}_{t \mid t-1}\right)$

$6 \quad \mathrm{P}_{t}=\left(\mathrm{I}-\mathrm{K}_{t} \mathrm{C}\right) \mathrm{P}_{t \mid t-1}$

7 where $\mathrm{A}$ is the constant velocity state transition model, $\mathrm{Q}$ is the process noise covariance, $\mathrm{R}$ is

8 the measurement noise covariance, $\mathrm{z}$ is the measurement vector consisting of the position and

9 size of the bounding box and $\mathrm{x}$ is the state vector consisting of the position and size of the

10 bounding box and their rates of change, $\mathrm{K}_{\mathrm{t}}$ is the optimal Kalman gain that minimises the

11 residual error, $\mathrm{I}$ is an identity matrix, $\mathrm{P}$ represents the error covariance matrix and $\mathrm{C}$ gives the

12 observation model.

13 To associate data from the R-CNN model and KF, the multi-object tracker accepts

14 detections from multiple sensors and assigns them to tracks using a global nearest neighbour

15 criterion. To ensure that the R-CNN and KF are assigned to the same track, the normalised

16 distance of the detections from the track must be less than a defined threshold. In the case of

17 discontinuous data, the tracks are deleted, and the procedure has to be reinitiated. These

18 discontinuities in the data need to be known beforehand as otherwise it will affect the generated

19 traffic conflict output. However, it is typically known during data collection that an error has

20 occurred.

21 The detection data is stored in a matrix in which the timestamp, x-position, y-position,

22 height and width of bounding box is given for each frame at $15 \mathrm{~Hz}$. From this data, the distance 
1 and the velocity of the detected vehicles can be estimated by using the Kalman filter predictions

2 and Inverse Perspective Transform (IPM) technique. The IPM technique is calibrated to

3 transform pixel coordinates from the image plane to the road plane. The transformation matrix

4 that maps points from the image plane to the road plane is defined by:

$5 \quad$ transformation matrix $=(\mathrm{D} *(\mathrm{~T} *(\mathrm{~B} * \mathrm{E})))^{-1}$

6 where $\mathrm{D}$ is the intrinsic matrix, $\mathrm{T}$ is the translation matrix, $\mathrm{B}$ is the rotation matrix and $\mathrm{E}$ is the

7 projection matrix of the camera. An estimate of the camera's intrinsic, extrinsic and the lens

8 distortion parameters is obtained by calibrating 20 images of a checkerboard calibration pattern

9 using the same camera used to collect data. The intrinsic parameters describe the internal

10 characteristics of the camera, such as the focal length of the lens, optical centre, and lens

11 distortion coefficients. These parameters help to improve image quality, correct for lens

12 distortion and map real-world distances to pixels. On the other hand, the extrinsic parameters

13 define the camera's position (translation) and angle (rotation) in space in relation to a fixed

14 object which is essential to structure from motion.

15 Therefore, by adopting this methodology of transforming the forward-facing image to a

16 'bird's eye view' image, the perspective distortion of the road surface and the non-linearity of

17 distances is eliminated. In order to compute the distance, the coordinates at the bottom edge of

18 the bounding box (which is the region where the vehicle touches the ground) are transformed

19 from the image plane to the road plane using the IPM.

While the states of the KF include the rate of change of position and size of the bounding

21 box, the velocity is not readily obtainable since the states cannot be converted to the closure rate

22 in meters per second. Instead, the states defining the rate of change are used to predict the 
1 position of the bounding box after 1 millisecond. Therefore, the current vehicle position and the

2 predicted vehicle position are converted to the road plane and are used to calculate the velocity

3 and acceleration based on the time difference between the current position and the predicted one.

4 Additionally, the bird's eye view images are used to identify sudden or unexpected lane

5 changes (LCs). In a bird's eye view, lane markers appear parallel and of uniform thickness

6 making it easier to detect. These images are converted to grayscale and then filtered with a ridge

7 detector. The resulting binary image indicated pixels belonging to the lane markers. A random

8 sample consensus (RANSAC) algorithm was then used to robustly generate candidate second

9 order polynomials that characterize the apparent lines in the binary image.

10 From the resulting information obtained from the images, an automated video analysis

11 system was developed to identify potential traffic conflicts associated with LC and rear-end (RE)

12 conflicts. These conflicts are generally identified through response to an evasive action, and the

13 temporal and/or spatial proximity (Zheng et al., 2014). The determining factors to develop this

14 system were based on the following criteria: time, distance, speed, braking, acceleration and

15 deceleration.

\section{$16 \quad$ 4.1.1 Lane change conflicts}

17 A LC conflict can result in either a sideswipe conflict or a RE conflict. If a Lane Changing

18 Vehicle (LCV) and the EV maintain their velocity and the traffic conflict angle is between $\left[5^{\circ}\right.$,

$1985^{\circ}$ ], this would result in a potential sideswipe conflict. However, when the traffic conflict angle

20 occurs between $\left[0^{\circ}, 5^{\circ}\right.$, the LC conflict is instead considered as a RE conflict (Kusano et al.,

21 2014) and the criteria defined for RE conflicts are applied. Moreover, a LC conflict exists

22 provided that the lateral acceleration of the $\mathrm{EV}$ is less than $0.07 \mathrm{~g}$. This threshold guarantees that

23 the EV doesn't change lane when it identifies a cut-in vehicle (Yang et al., 2019) or that it not an 
1 overtaking manoeuvre by the EV (Chen et al., 2015). Therefore, a potential LC traffic conflict is

2 identified if:

a) the total time headway between the LCV and the EV is less than $3 \mathrm{~s}$, plus the time for the LCV to enter the EV's lane which can be calculated by: $t_{L C}=l_{L C V} / V_{L C V}(\mathrm{Qu}$ et al., 2014) where $l_{L C V}$ and $V_{L C V}$ is the length and velocity of the LCV. Since TTC is later used as one of the factors in the DNN model, time headway is instead employed to identify conflicts as TTC and time headway are considered independent of each other (Vogel, 2003).

b) the lateral distance between $\mathrm{LCV}$ and $\mathrm{EV}$ is greater than $1.5 \mathrm{~m}$ and less than $2.2 \mathrm{~m}$ showing that the LCV intends to start its movement to the EV's lane (Yang et al., 2019) while the EV is accelerating longitudinally or moving with constant velocity.

c) the longitudinal available space is less than the maximum longitudinal distance of $75 \mathrm{~m}$ between the LCV and the EV. This is because this distance is appropriate to determine when two vehicles are interacting (Bham, 2009; Yang et al., 2019).

d) the rate of change of speed when the LCV initiates the LC manoeuvre till it stabilises within the boundaries of the EV's lanes is greater than $20 \%$ in absolute value. This is because it implies that an unstable deceleration and/or acceleration is taking place which can suggest an adverse impact on the EV (Yang et al., 2019).

\subsubsection{Rear-End Conflicts}

A RE conflict takes place when the EV nearly collides with a vehicle in its forward pathway provided that the traffic angle conflict occurs between $\left[0^{\circ}, 5^{\circ}\right]$. From the video analysis system, a RE traffic conflict is highlighted if:

a) the time headway is less than $3 \mathrm{~s}$ when the lateral distance is less than $2 \mathrm{~m}$ (Kusano et al., 2014) and the lateral acceleration is less than $0.07 \mathrm{~g}$. The lateral distance of $2 \mathrm{~m}$ is used even 
though it is slightly larger than half the lane width to allow for flexibility in identifying preceding vehicles while the lateral acceleration confirms that the $\mathrm{EV}$ is not changing lane.

b) the speed of the EV is greater than the speed of the PV during deceleration of the PV, and whilst the EV is accelerating or moving at a constant velocity.

c) the speed of the PV is greater than the speed of the EV, but the PV is decelerating and the $\mathrm{EV}$ is accelerating or moving at constant velocity.

d) the PV exceeds its threshold for a braking event. This is set at $0.3 \mathrm{~g}$ which results in acceleration or deceleration of $\pm 2.943 \mathrm{~m} / \mathrm{s}^{2}$ respectively. If this is exceeded, a traffic conflict is identified. This threshold value is also adopted in Naito et al., (2009) and Miyajima et al., (2011).

e) the EV brakes and its speed is greater than $10 \mathrm{mph}$ for at least $1 \mathrm{~s}$ of the braking time. This ensures that it is not continuously braking due to a congested environment.

Merging and diverging areas are also considered in this study. The most common conflicts occurring in these areas are also RE and LC (Wang et al., 2017). As a result, the same criteria developed for both RE and LC conflicts are adopted when a merging or diverging section is identified.

The development of this video data analysis system accelerated the procedure and ensured that any errors associated with human observation in the first stage of the conflict identification system were eliminated. Based on these criteria, each traffic conflict identification was validated by manually observing the corresponding videos collected from the forward-facing camera. As a result, a significant amount of time was saved as the points observed were only those identified and highlighted as potential traffic conflicts by the system. During manual observations, some of the events were misclassified and not included to prevent bias in the results. 


\section{$1 \quad 4.2 \quad$ Classifier development}

2 Following the identification of traffic conflicts, a DNN model is developed to predict traffic

3 conflicts based on a set of influencing factors. For the development of the DNN, it requires both

4 the output (i.e. tracked vehicles which are classified as 'threats' as discussed in the previous

5 section) and the input (corresponding estimated SSMs and traffic variables) for the same

6 timestamp. These are fed into the DNN network to train and identify patterns of what arises from

7 the factors to result in a traffic conflict.

8 While the input and output layers of the network can be directly accessed, the hidden

9 layers made up of a number of neurons, work as a black box. These layers are connected together

10 utilising weights and biases. Indeed, different hidden layers with a different number of nodes are

11 employed in order to structure DNN models for each activation function. Two different nonlinear

12 activation functions are adopted throughout the network to train the model: tanh and maxout.

13 The tanh activation function is a rescaled and shifted logistic function known for its symmetry

14 about zero, and so the training also converges faster. It is formulated as:

$15 \tanh (\mathrm{x})=\frac{2}{1+\mathrm{e}^{-2 \mathrm{x}}}-1$

16 while the maxout function is a generalisation of the Rectified Linear activation in which each

17 neuron picks the most significant output of separate channels and each channel has its own

18 weights and bias values, is formulated as:

$19 \operatorname{maxout}\left(\mathrm{x}_{1}, \mathrm{x}_{2}\right)=\max \left(\mathrm{x}_{1}, \mathrm{x}_{2}\right)$

20 Therefore, the nonlinear transformation is carried out by these functions. The data is transferred

21 throughout the hidden layers and the output from each neuron $p$ the hidden layer is obtained as:

$22 \quad \mathrm{H}_{l p}=\mathrm{f}_{l p}\left(\sum_{q=1}^{\mathrm{n}_{1-1}} \mathrm{w}_{l p q}\left(\mathrm{H}_{(l-1) q}\right)+\mathrm{b}_{l p}\right)$ 
1 where $\mathrm{H}_{l p}$ refers to the output from hidden layer $l$ and neuron $p, \mathrm{f}_{l p}$ is the activation function for

2 layer $l$ and particular neuron $p, \mathrm{w}_{l p q}$ is the weight coefficient for the $q^{t h}$ input for layer $l$ and

3 neuron $p$, and $\mathrm{b}_{l p}$ is the bias parameter for layer $l$ and neuron $p$. Both the number of hidden

4 layers and neurons are varied to identify the optimal output $\mathrm{y}_{O}$ from the output layer $O$, given as:

$5 \quad \mathrm{y}_{O}=\mathrm{f}_{O}\left(\sum_{q=1}^{n_{t}} \mathrm{w}_{O q}\left(\mathrm{H}_{O q}\right)+\mathrm{b}_{O}\right)$

6 where $\mathrm{f}_{O}$ is the softmax activation function, $\mathrm{w}_{O q}$ and $\mathrm{b}_{O}$ are weights and bias in the output layer

7 and $n_{t}$ is the total number of neurons in final hidden layer $\mathrm{H}_{O}$. Nevertheless, when the data is

8 imbalanced and a classification is performed, the cross entropy loss function is optimised by the

9 model and is given as (Candel and Parmar, 2015):

$10 \mathrm{~L}(k)=-\sum_{\mathrm{y} \in\{0,1\}}\left(\mathrm{O}_{\hat{y}}(k) * \ln \left(\mathrm{O}_{y}(k)\right)+\left(1-\mathrm{O}_{\hat{y}}(k)\right) * \ln \left(1-\mathrm{O}_{y}(k)\right)\right)$

11 where $\mathrm{O}_{y}(k)$ and $\mathrm{O}_{\hat{y}}(k)$ are the actual and predicted output respectively, from output layer $\mathrm{O}$ and

12 iteration $k$. Since big data is used in the study, overfitting can become a problem hence

13 regularisation techniques can be adopted. This is done either by changing the Lasso

14 Regularisation (L1) or Ridge Regularisation (L2) in order to modify the loss function, or by

15 applying the dropout technique in which the network is forced to learn multiple independent

16 representations of the data.

17 In order to evaluate the performance of the DNN model, $\mathrm{K}$-fold cross-validation is a

18 commonly used technique (Rodríguez et al., 2010). For instance, a 10-fold cross-validation

19 means that the data is divided into 10 -folds. The total number of iterations are equal to the

20 number of folds, and while keeping one-fold for validation, the remaining folds are used for

21 training. The AUC values give the estimation resulting from each fold. Moreover, the

22 performance of the DNN model can be evaluated based on the following criteria where TP is

23 equivalent to true positive, $\mathrm{FN}$ is false negative, $\mathrm{FP}$ is false positive, and TN is true negative: 
1 1. Sensitivity - This variable gives the prediction accuracy of how many conditions are

2 predicted as traffic conflicts out of all the present traffic conflicts.

$3 \quad$ Sensitivity $=\frac{\mathrm{TP}}{\mathrm{TP}+\mathrm{FN}}$

4 2. Precision - This variable shows how many conditions are predicted as traffic conflicts, out $5 \quad$ of all the predicted traffic conflicts. Precision $=\frac{\mathrm{TP}}{\mathrm{TP}+\mathrm{FP}}$

7 3. False alarm rates - This represents the number of safe conditions predicted as traffic $8 \quad$ conflicts.

$9 \quad \mathrm{FAR}=\frac{\mathrm{FP}}{\mathrm{FP}+\mathrm{TN}}$

10 4. Accuracy - This shows if both 'safe traffic dynamics' and 'traffic conflict' cases are 11 predicted correctly.

12 Accuracy $=\frac{\mathrm{TP}+\mathrm{TN}}{\mathrm{TP}+\mathrm{FN}+\mathrm{FP}+\mathrm{TN}}$

13 5. Area Under Curve values - This variable gives the predictive performance of the model.

14 It usually ranges between $0-1$ and the higher the AUC value, the better the performance.

15 Additionally, several methods are available to construct a scoring system for variable importance

16 such that the most influencing factors which contributed to a traffic conflict detection can be

17 deduced. For the DNNs developed in this paper, the methodology developed by Gedeon (1997)

18 is applied and the basis of these scores are the network's connection weights. 


\section{$2 \quad 5.1 \quad$ Data Integration Architecture}

3 The data integration architecture developed in this study offers a system to integrate data from

4 multiple sensors in real-time in a heterogeneous environment. In fact, this architecture provides a

5 reliable storage to access and extract meaningful information from a big dataset and

6 communicate findings of several factors in a timely manner.

7 A unique feature of this analysis is that it is able to show how the different parameters estimated

8 vary with other variables. More specifically, three examples are presented of how SSMs vary

9 with traffic variables in Figure 4.

a. Flow against Speed by lane

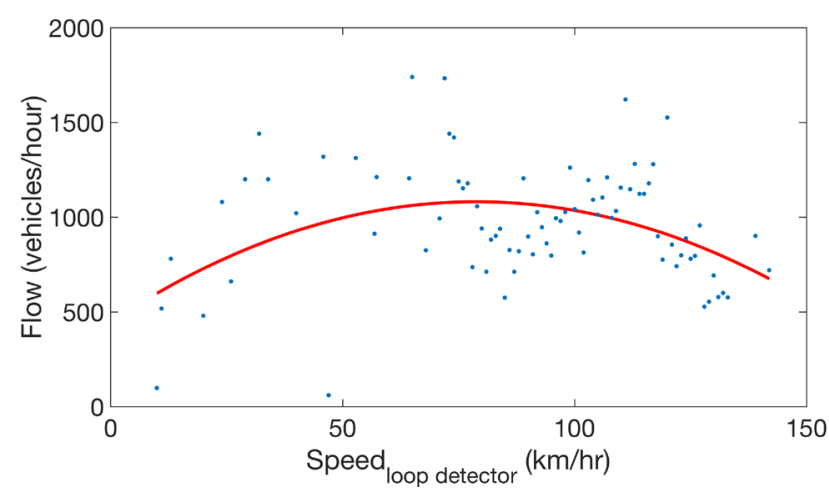

c. 1/DRAC against Density

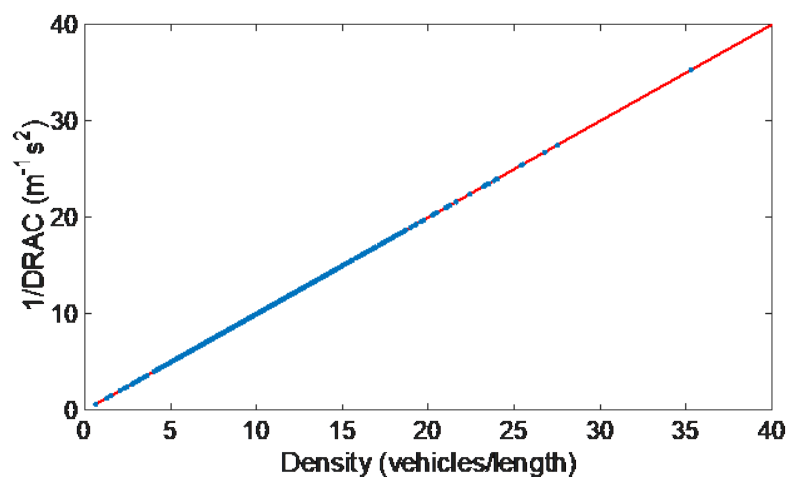

b. TTC against Speed from the ego-vehicle

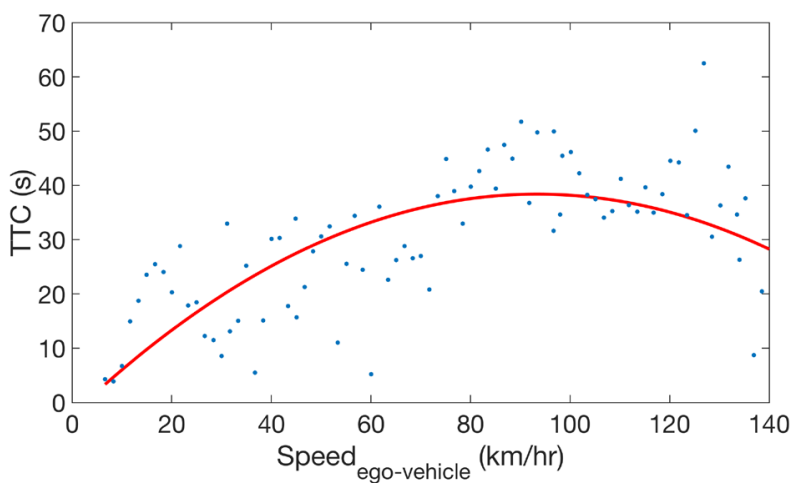

d. PSD against Flow

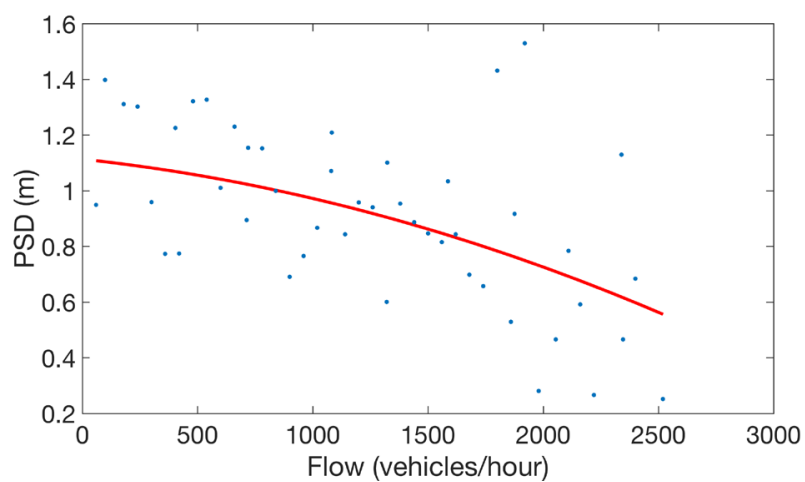

11 Fig. 4. The variation of factors with the same timestamp 
1 These results clearly show that a relationship exists between traffic variables and SSMs. A

2 different property-based SSM (time, distance and deceleration) is tested, and in each case, an

3 association can be identified. In fact, in Figure 4(a) the general relationship is obtained between

4 flow and the speed, Figure 4(b) shows that an inverse quadratic relationship exists between TTC

5 and ego-vehicle speed, Figure 4(c) shows a linear relationship such that as the density increases

6 the deceleration decreases and Figure 4(d) shows that as the flow increases hence there is less

7 space on the road the PSD value decreases as well. This highlights the need for their effect to be

8 embedded in the DNN model to add insight in identifying traffic conflicts and improve the

9 predictability performance. Additionally, each SSM exhibits a varying relationship with a traffic

10 variable which suggests the need for adopting more than one SSM. Moreover, to test the

11 thresholds for each SSM found in the literature (see Table 1), from the data integration system,

12 the SSM distributions during safe traffic dynamics and traffic conflicts were extracted and are

13 presented in Figure 5.
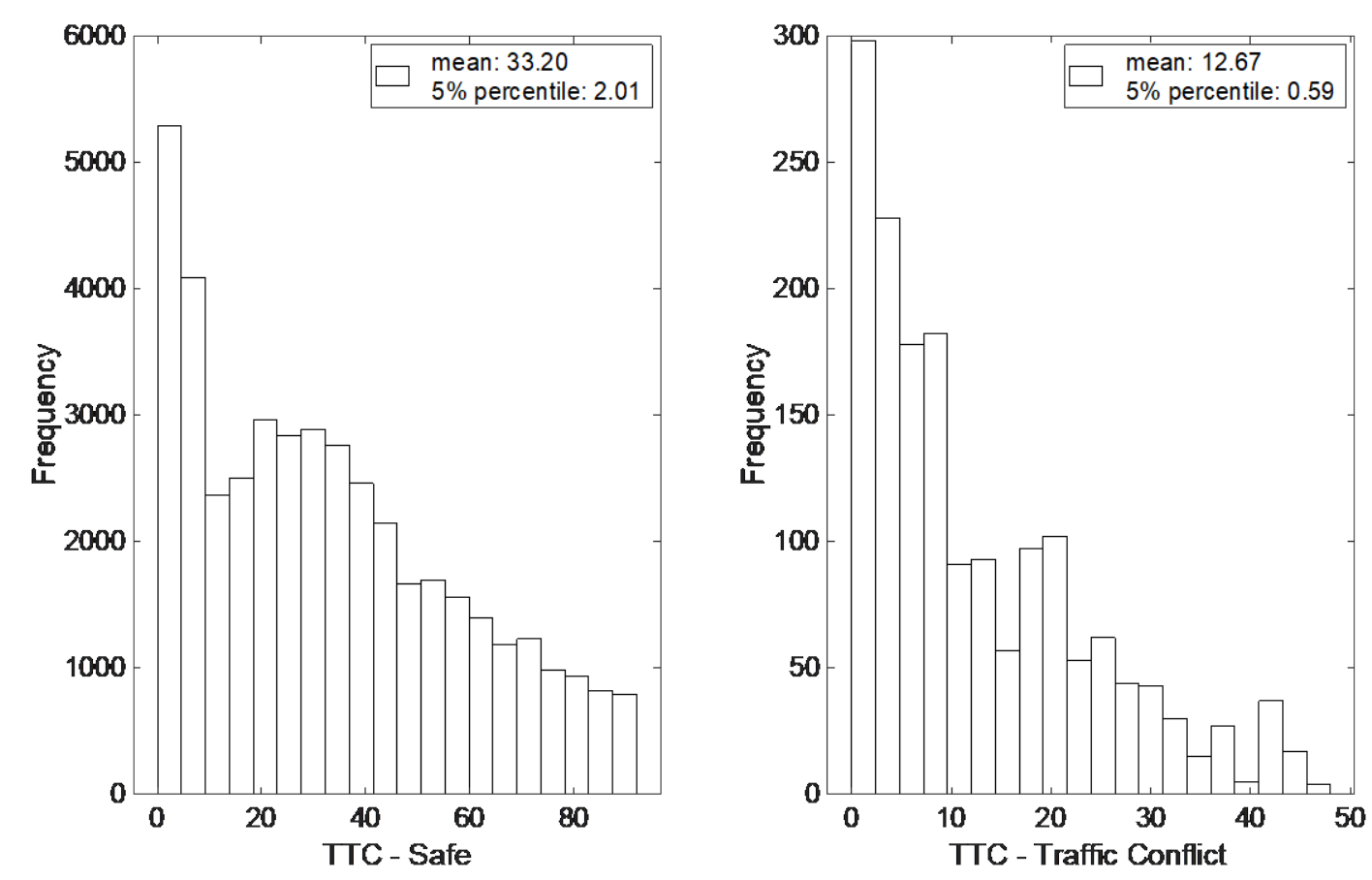

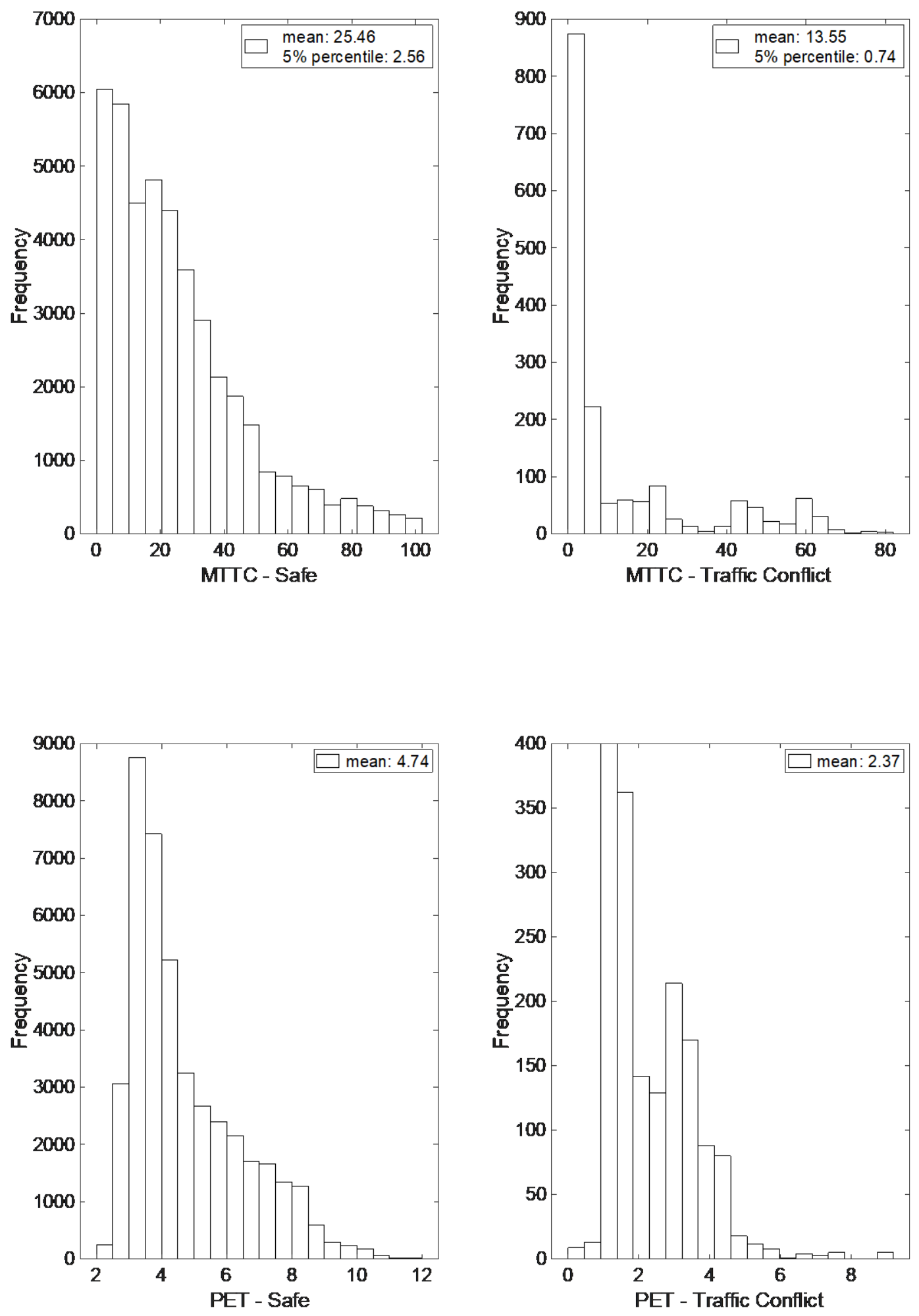

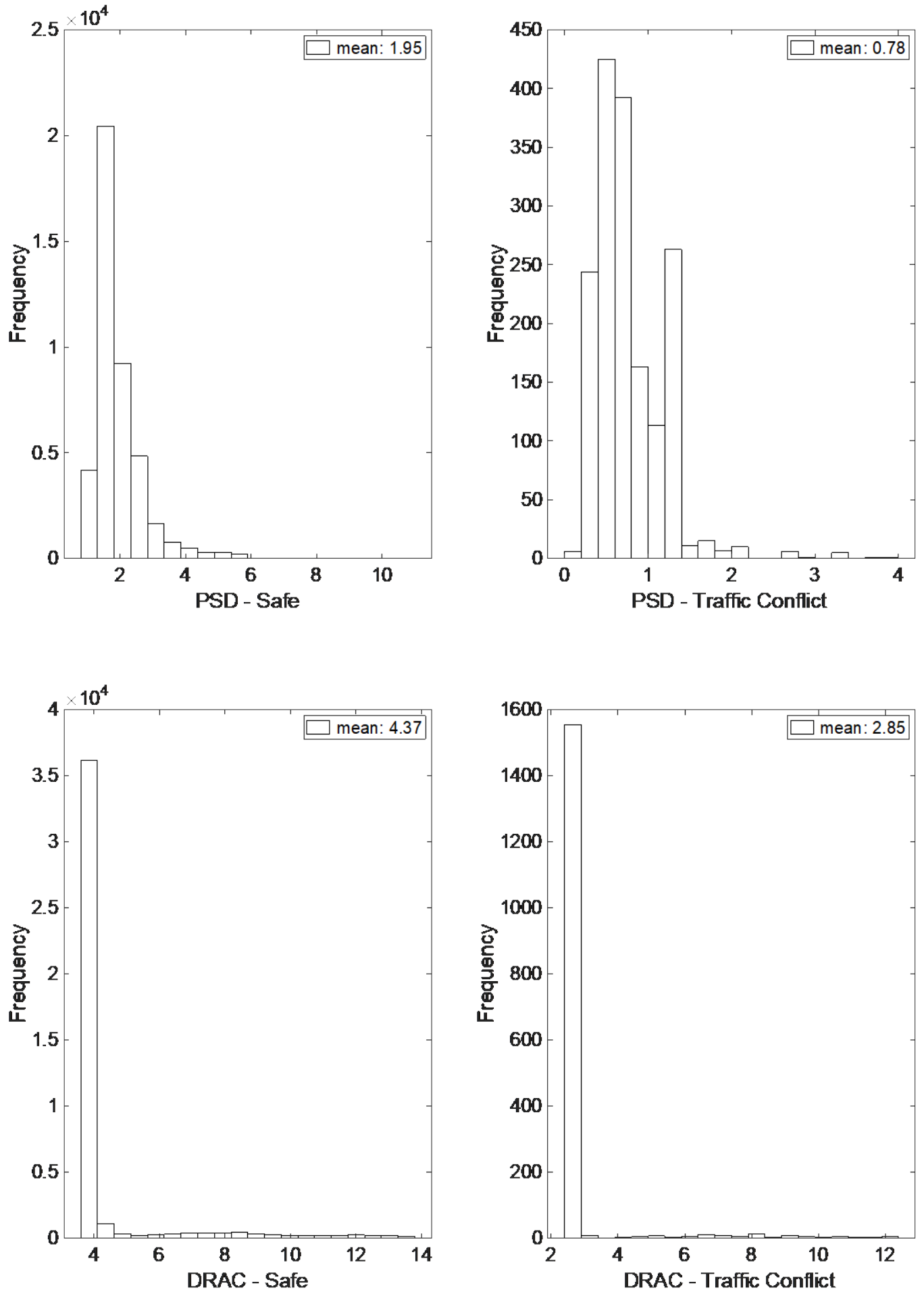

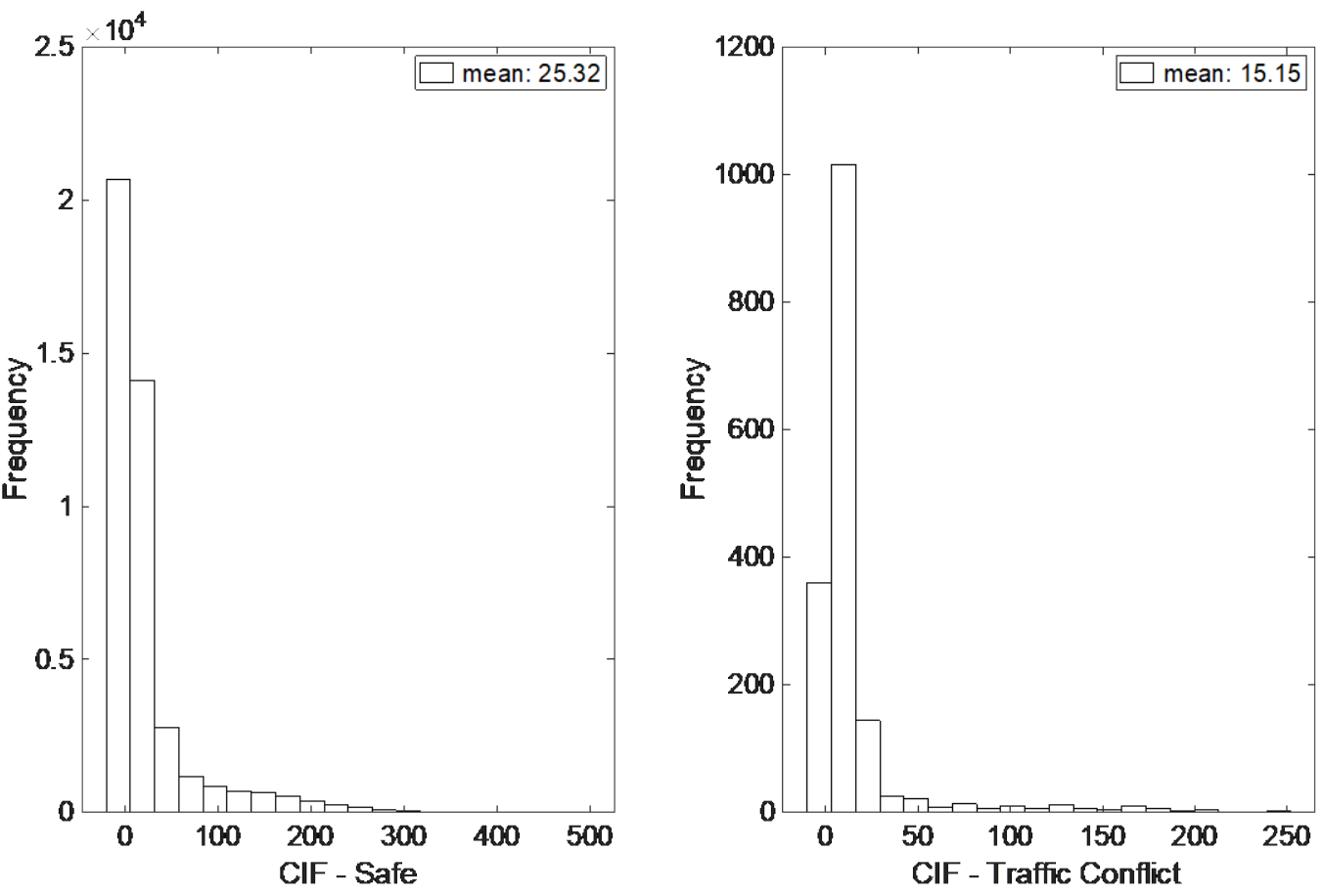

2 Fig. 5. The distribution for each SSM during safe and traffic conflicts

3 While some mean values are relatively higher than the thresholds defined for each of the SSMs

4 in Table xxx, it is important to note that these values represent averages over all the estimated

5 SSMs when a traffic conflict is identified based on the criteria, till when the detected vehicle no

6 longer posed a threat to the ego-vehicle. In particular, when considering TTC and MTTC, the $5^{\text {th }}$

7 percentile value is more representative than the mean value since the lower it is, the more unsafe

8 it is, therefore there is more interest in the left-hand side of the distribution (Papazikou et al.,

9 2019). These values also give a clear indication that the values of SSMs are always lower in a

10 traffic conflict condition relative to the 'safe' traffic conditions. This shows that their influence

11 as factors in the DNN model are beneficial since they would be able to add insight in predicting

12 traffic conflicts. 


\section{$1 \quad 5.2 \quad$ Evaluation of the R-CNN Model using Testing Data}

2 To evaluate the performance of the R-CNN model to identify vehicles in the images, training

3 was carried out on 4,200 images accounting for $70 \%$ of the data. Each image included the region

4 of interest for vehicles. For detection purposes, the input size of the image input layer was

5 chosen as $32 \times 32 \times 3$ since smaller portions of the image should be analysed. A total of 15 layers

6 were setup to develop the network in which every layer was trained in mini-batches of 128

7 images for 60 epochs using SGD with a learning rate of 0.0001 . In the evaluation process, the

8 detected bounding boxes that had a ratio of 0.7 overlap with ground-truth boxes, were identified

9 as correct while boxes with no overlap were identified as misdetection. Based on 1,800 images in

10 the testing data, the average precision of the network is 0.675 . This network was used throughout

11 for all the video data to identify vehicles and based on the criteria developed the conflicts were

12 identified surrounding the ego-vehicle.

\section{$13 \quad 5.3 \quad$ Real Time Traffic Conflict Detection Model}

14 A total of 26 variables were used as input variables to the DNN model consisting of all the six

15 SSMs presented in Table 1, the ego-vehicle's speed, speed variance between lanes, lane number,

16 traffic variables by lane (i.e. speed, density, flow, headway, occupancy) and their mean and the

17 standard deviations across all lanes. The input variables of the DNN model have been fine-tuned

18 with maximum effort by considering the weighting factor between the layers during the training

19 process of multiple DNN models. The variables with consistently high weighting were the only

20 used variables to develop the predictor. The output variables extracted from the video data

21 consisted of 42,541 safe traffic dynamics and 1,663 traffic conflicts. The dataset was then

22 randomly partitioned into $70 \%-30 \%$ for training and testing data respectively as it provides the 
1 best trade-off. As a result, the training data consisted of 29,785 safe conditions and 1,158 traffic

2 conflicts while the testing data consisted of 12,756 safe conditions and 505 traffic conflicts.

3 The DNN model was developed by testing two of the activation functions: tanh and

4 maxout, and by varying the number of hidden layers i.e. 2, 3, 4, 5 layers, and the number of

5 nodes within the hidden layer i.e. $10,12,15,18,20,25,30$ resulting in $28 \mathrm{DNN}$ modes for each

6 activation function. The best 4 models are presented in Table 4 together with their performance

7 metrics for each DNN model.

$8 \quad$ Table 4

9 Performance metrics in the DNN model

\begin{tabular}{|c|c|c|c|c|c|c|c|c|c|}
\hline \multirow[b]{2}{*}{ No } & \multirow{2}{*}{$\begin{array}{l}\text { Activa- } \\
\text { tion }\end{array}$} & \multirow{2}{*}{$\begin{array}{l}\text { Hidden } \\
\text { layers }\end{array}$} & \multirow{2}{*}{$\begin{array}{c}\text { Train } \\
\text { AUC }\end{array}$} & \multirow{2}{*}{$\frac{\text { Validation }}{\text { AUC }}$} & \multicolumn{5}{|c|}{ Test Data } \\
\hline & & & & & $\begin{array}{c}\text { Sensiti } \\
\text { vity }\end{array}$ & $\begin{array}{l}\text { False alarm } \\
\text { Rate (FAR) }\end{array}$ & Precision & Accuracy & AUC \\
\hline \multirow{6}{*}{1} & \multirow{6}{*}{ Tanh } & \multirow{6}{*}{$\begin{array}{c}{[30,30,} \\
30, \\
30]\end{array}$} & \multirow{6}{*}{0.940} & \multirow{6}{*}{$\begin{array}{c}0.938 \\
\text { s.d(0.0301) }\end{array}$} & $66.7 \%$ & $3.0 \%$ & $47.2 \%$ & 0.959 & \multirow{6}{*}{0.935} \\
\hline & & & & & $70.5 \%$ & $5.0 \%$ & $36.0 \%$ & 0.941 & \\
\hline & & & & & $75.4 \%$ & $7.0 \%$ & $30.0 \%$ & 0.924 & \\
\hline & & & & & $78.8 \%$ & $10.0 \%$ & $23.5 \%$ & 0.895 & \\
\hline & & & & & $89.7 \%$ & $20.0 \%$ & $14.7 \%$ & 0.795 & \\
\hline & & & & & $92.8 \%$ & $30.0 \%$ & $11.3 \%$ & 0.709 & \\
\hline \multirow{6}{*}{2} & \multirow{6}{*}{ Tanh } & \multirow{6}{*}{$\begin{array}{c}{[18,} \\
18,18, \\
18]\end{array}$} & \multirow{6}{*}{0.936} & \multirow{6}{*}{$\begin{array}{c}0.930 \\
\text { s.d(0.0099) }\end{array}$} & $64.0 \%$ & $3.0 \%$ & $46.1 \%$ & 0.958 & \multirow{6}{*}{0.922} \\
\hline & & & & & $68.3 \%$ & $5.0 \%$ & $35.2 \%$ & 0.940 & \\
\hline & & & & & $68.7 \%$ & $7.0 \%$ & $27.9 \%$ & 0.921 & \\
\hline & & & & & $78.6 \%$ & $10.0 \%$ & $23.5 \%$ & 0.895 & \\
\hline & & & & & $85.9 \%$ & $20.0 \%$ & $14.5 \%$ & 0.801 & \\
\hline & & & & & $91.7 \%$ & $30 \%$ & $10.8 \%$ & 0.709 & \\
\hline \multirow{6}{*}{3} & \multirow{6}{*}{ Maxout } & \multirow{6}{*}[20,20]{} & \multirow{6}{*}{0.936} & \multirow{6}{*}{$\begin{array}{c}0.931 \\
\text { s.d(0.0186) }\end{array}$} & $67.3 \%$ & $3.0 \%$ & $48.2 \%$ & 0.960 & \multirow{6}{*}{0.927} \\
\hline & & & & & $77.8 \%$ & $5.0 \%$ & $37.8 \%$ & 0.943 & \\
\hline & & & & & $79.2 \%$ & $7.0 \%$ & $31.2 \%$ & 0.926 & \\
\hline & & & & & $83.1 \%$ & $10.0 \%$ & $24.8 \%$ & 0.897 & \\
\hline & & & & & $91.5 \%$ & $20.0 \%$ & $15.1 \%$ & 0.804 & \\
\hline & & & & & $94.3 \%$ & $30.0 \%$ & $10.9 \%$ & 0.709 & \\
\hline \multirow{5}{*}{4} & \multirow{5}{*}{ Maxout } & \multirow{5}{*}[15,15]{} & \multirow{5}{*}{0.939} & \multirow{5}{*}{$\begin{array}{c}0.931 \\
\text { s.d( }(0.0186)\end{array}$} & $63.4 \%$ & $3.0 \%$ & $46.0 \%$ & 0.958 & \multirow{5}{*}{0.913} \\
\hline & & & & & $72.1 \%$ & $5.0 \%$ & $37.6 \%$ & 0.943 & \\
\hline & & & & & $75.0 \%$ & $7.0 \%$ & $30.7 \%$ & 0.925 & \\
\hline & & & & & $78.6 \%$ & $10.0 \%$ & $24.1 \%$ & 0.896 & \\
\hline & & & & & $90.7 \%$ & $20.0 \%$ & $15.3 \%$ & 0.806 & \\
\hline
\end{tabular}




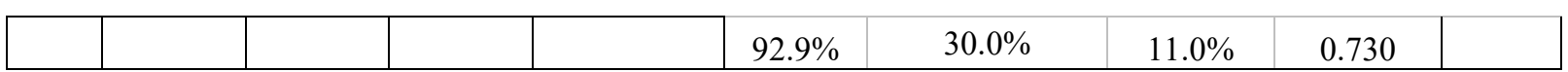

2 The AUC values based on the test data presented in this Table, resulted in four high values

3 indicating that the DNN network can provide a good prediction performance. By comparing the

4 test data AUC values for each model, Model 1 has the best performance. This model also

5 achieved the highest AUC for the training, validation and testing data. The AUC values for all 4

6 models are all relatively high which shows that this methodology achieves good classification

7 and has a strong prediction ability. In addition, based on Model 1, the most influential factor was

8 found out to be: traffic flow followed by speed variance between the lanes while the most

9 influential SSM was distance-based PSD. Furthermore, from Figure 6, the ROC curves for each

10 model rise rapidly towards the upper-left-hand corner of the graph which reflects the good

11 performance of each model tested. 
1. $\operatorname{Tanh}[30,30,30,30]$

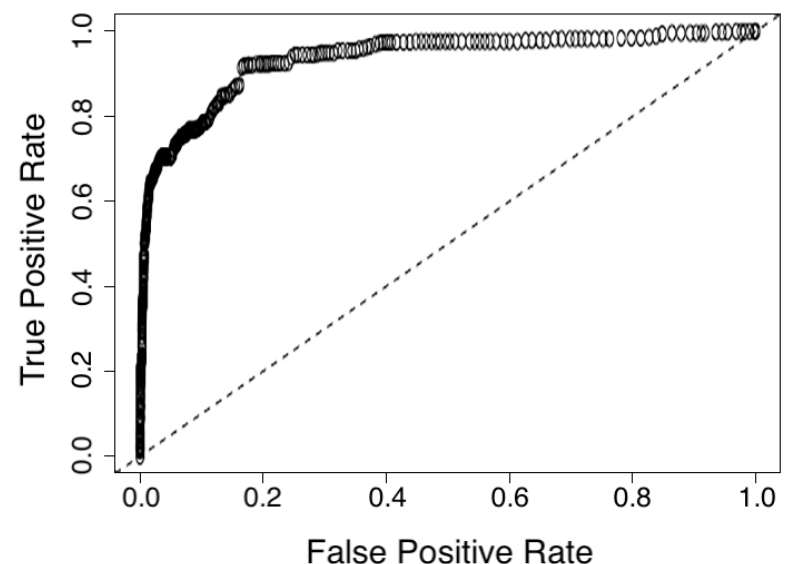

3. Maxout $[20,20]$

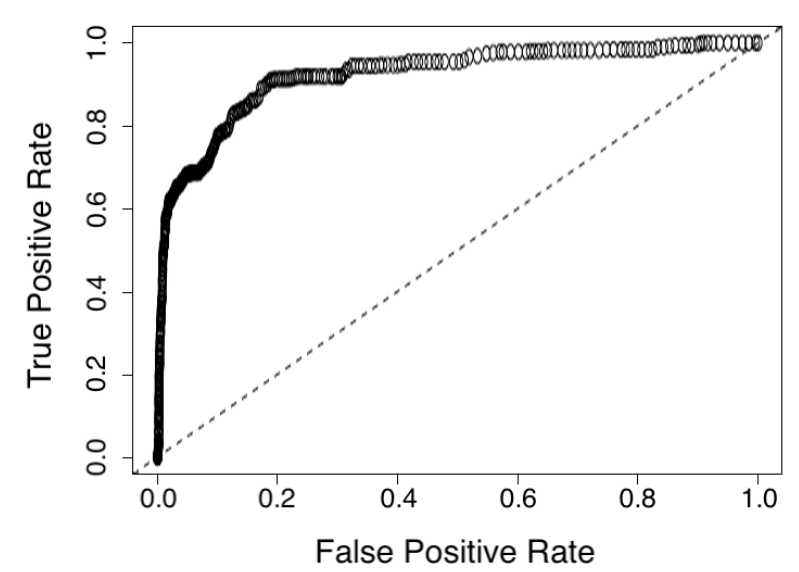

2. Tanh $[18,18,18]$

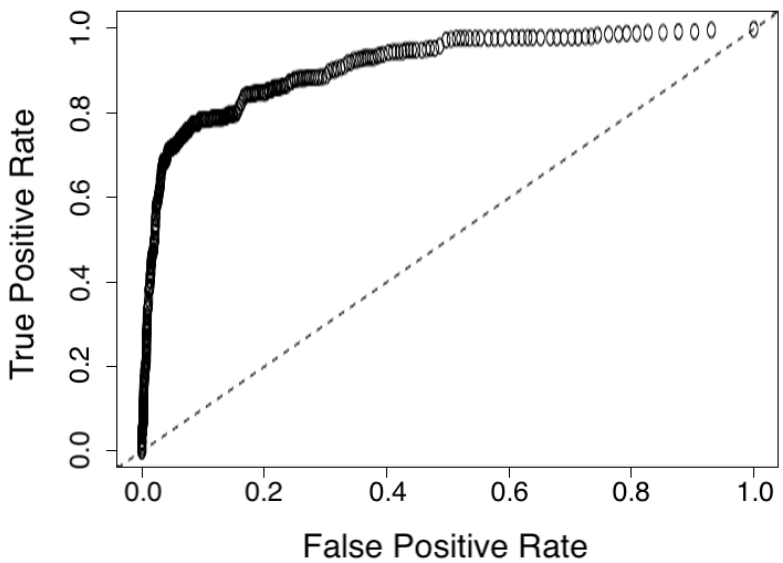

4. Maxout $[15,15]$

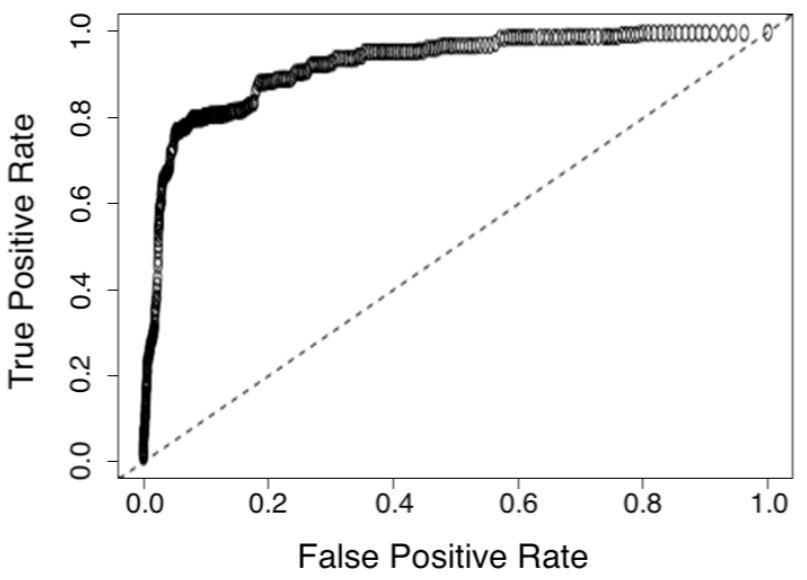

\section{Fig. 6. Test Data ROC curves from DNN model}

3 To further test the prediction accuracy of the DNN models, six false alarm rates (FAR) were

4 determined for each model $(3 \%, 5 \%, 7 \%, 10 \%, 20 \%$ and $30 \%)$ in order to determine the

5 sensitivity of the model. Results show that Model 3 was the most sensitive model and consisted

6 of 2 hidden layers, 20 nodes in each layer and the maxout as the activation function. In fact, it

7 can predict $78 \%$ and $80 \%$ of the traffic conflicts at the cost of $5 \%$ and $10 \%$ false alarm rate

8 respectively. 
2 From the $50 \mathrm{DNN}$ models tested with a varying number of hidden layers, nodes, and two

3 different activation functions, the best 4 models which achieved the highest AUC value with the

4 testing data were presented. The model with the highest prediction for traffic conflicts consists of

54 hidden layers, 30 nodes in each layer and the $\tanh$ as the activation function. The model

6 performance was also evaluated via 10-fold cross-validation. The results show that the AUC

7 values obtained 0.935 on the testing data in this study. This exceeds that of previous work when

8 using other machine learning classifiers which only achieved accuracies of up to 0.834 (Yang et

9 al., 2017). This is because some of these previous studies employ machine learning predictors

10 which cannot be used for highly imbalanced data sets. Therefore, some of their data may have

11 been omitted which can affect the calibration of the model and therefore a high prediction

12 accuracy is not achieved. As a result, by making use of the DNN network while considering a

13 loss function which caters for highly imbalanced data sets (Wang et al., 2016b), more accurate

14 predictions are obtained. In this research the 'Mean Squared False Error' loss function was

15 adopted because of its ability to capture classification errors from both the majority and minority

16 classes equally and this shows in the prediction results obtained.

17 A real-time implementation of a model presented in this paper is in an ADAS system to

18 warn drivers of any potential traffic conflicts. Moreover, if vehicles are connected with the

19 ability to share information (e.g. platooning), this model can provide a coordinated traffic

20 conflict prediction such that not only the ego-vehicle is controlled, but also the preceding

21 vehicle. It is essential that the signals in proactive safety systems are released with enough time

22 for the driver to react and avoid these conflicts but not too hasty or too recurrent to become an

23 annoyance or interference to the driver. However, if the FAR is set too low so that the driver 
1 does not become immune to the warnings, the prediction performance is very low. Nevertheless,

2 the prediction accuracy in this study from the ROC curves is relatively high and for an accepting

3 FAR of $5 \%, 71 \%$ of traffic conflicts can be predicted. If a threshold is selected to accept $10 \%$

4 FAR, the prediction accuracy increases and around $78 \%$ of the traffic conflicts are identified.

5 This performance is sufficient for implementation in a real-time warning technology based on

6 the models presented in this paper (Yu and Abdel-Aty, 2014).

7 Moreover, these sensitivity results also show that these models are more sensitive than

8 previous studies which underline the identification ability in real-time traffic conflicts prediction

9 that the DNN technique has. However, the most sensitive model found from the 4 best models is

10 not the same one which obtained the highest AUC for the testing data. In fact, Model 3 was the

11 most sensitive model given that for a $10 \%$ FAR it is able to predict $83 \%$ of traffic conflicts.

12 When developing the dataset for this study, traffic conflicts were classified by image

13 processing techniques including an R-CNN network to identify the vehicles. This was carried out

14 to create an output variable to correspond to the input data from which the DNN model can learn.

15 However, the precision of the R-CNN network can be further improved by modifying its

16 parameters, adding more layers and more training data on which the network can learn from.

17 Moreover, a Faster R-CNN can be employed to further enhance the knowledge gained from the

18 images and obtain more reliable traffic conflicts data and avoid any erroneous data. Previous studies have confirmed that the real-time prediction models cannot be directly

20 transferred from one road to another because of the variations in traffic patterns and driver

21 population. Since this DNN model is based on motorway traffic readings, this model cannot be

22 used for an urban setting. However, transferability can be possible for roadways with similar 
1 roads such as other motorways. Furthermore, the methodology can be applied to any type of road

2 network.

3 While the proposed DNN outperformed other machine learning classifiers, there are some

4 limitations in the study such as the lack of detail in the data namely the weather conditions,

5 drivers, road design which can improve the performance of the DNN. These added variables

6 could be beneficial for the model to improve the prediction accuracy further since they can

7 characterise a traffic condition. Moreover, in this work 50,000 data points were dealt with and by

8 increasing the size of the data set the predictability of these models is expected to increase.

9 Therefore, future work will include more testing especially in different weather conditions, to

10 further assess the performance of the model and tune it accordingly as well as testing the

11 predictive performance of the DNN model on a validation model.

12 7. Conclusion

13 This paper presented a new model for predicting traffic conflicts by using deep learning. A

14 centralised digital architecture was developed to integrate large volume and heterodox in-vehicle

15 sensors data with highly disaggregated traffic data. From this architecture, it was possible to

16 develop relationships between different surrogate safety measures (SSMs) derived from in-

17 vehicle sensors data and traffic variables such as speed, density and flow. For instance, time-to-

18 collision (TTC) - an SSM varies with the mean travelling speed indicating that a single threshold

19 value of TTC may not be appropriate for detecting a potential collision at different speeds. An

20 image processing technique aided by an R-CNN was employed to identify all the vehicles with

21 respect to the ego-vehicle from the video data, and several criteria were formulated to generate

22 the output variable for each input. The output variable was able to distinguish between traffic

23 conflicts and safe road dynamics which were subsequently integrated with the SSMs and the 
1 traffic variables to develop the DNN model. From the developed DNN model continuously

2 running at $15 \mathrm{~Hz}$, real-time predictions of traffic conflicts were achieved because of its capability

3 to be applied on a highly imbalanced dataset.

4 Real-time traffic conflict prediction model offered a high prediction, accuracy, sensitivity

5 and a low false alarm rate. In fact, results show that the DNN network can predict $71 \%$ and $78 \%$

6 of the traffic conflicts at the cost of 5\% and $10 \%$ false alarm rate respectively.

7 Moreover, the results show that the best DNN model provides an accuracy of $94 \%$ and as

8 a result, have the potential to be used in ADAS to develop proactive safety management

9 strategies for improving traffic safety. Furthermore, if vehicles are connected, active safety

10 features (e.g. early prediction of traffic conflicts) can be shared between these vehicles and

11 therefore the risk of traffic collisions are mitigated. 


\section{References}

2 Abdel-Aty, M., Uddin, N., Pande, A., 2005. Split Models for Predicting Multivehicle Crashes

3 During High-Speed and Low-Speed Operating Conditions on Freeways. Transp. Res. Rec.

$4 \quad$ J. Transp. Res. Board 1908.

5 Archer, J., 2005. Indicators for traffic safety assessment and prediction and their application in 6 micro-simulation modelling: A study of urban and suburban intersections. Acad. thesis, R.

7 Inst. Technol. Dep. Infrastructure, Div. Transp. Logist. Cent. Transp. Res. Royal Institute of 8 Technology, Stockholm, Sweden.

9 Bham, G., 2009. Estimating Driver Mandatory Lane Change Behavior on a Multi lane Freeway.

10 Transp. Res. Board 88th Annu. Meet. 573 .

11 Candel, A., Parmar, V., 2015. Deep Learning with H2O Deep Learning with H2O September .

12 Chan, C.-Y., 2006. Defining Safety Performance Measures of Driver-Assistance Systems for

13 Intersection Left-Turn Conflicts. Intell. Veh. Symp. (IV), IEEE 25-30.

14 Chen, R., Kusano, K.D., Gabler, H.C., 2015. Driver behaviour during lane change from the 10015 car naturalistic driving study. Traffic Inj. Prev. 16, 1-10.

16 Gedeon, T.D., 1997. Data Mining of Inputs: Analysing Magnitude and Functional Measures. Int.

17 J. Neural Syst. 08 02, 209-218.

18 Guido, G., Saccomanno, F., 2010. Comparing safety performance measures obtained from video 19 capture data. J. Transp. Eng. 137 July , 481-491.

20 He, H., Garcia, E.A., 2009. Learning from imbalanced data. IEEE Trans. Knowl. Data Eng. 219 
$1 \quad, 1263-1284$.

2 Hossain, M., Muromachi, Y., 2013. Understanding crash mechanism on urban expressways

3 using high-resolution traffic data. Accid. Anal. Prev. 57, 17-29.

4 Katrakazas, C., Quddus, M.A., Chen, W.-H., 2017. A new methodology for collision risk

5 assessment of autonomous vehicles. Transp. Res. Board 96th Annu. Meet.

6 Kusano, K.D., Montgomery, J., Gabler, H.C., 2014. Methodology for identifying car following 7 events from naturalistic data. IEEE Intell. Veh. Symp. Proc. Iv , 281-285.

8 Laureshyn, A., Svensson, Å., Hydén, C., 2010. Evaluation of traffic safety, based on micro-level 9 behavioural data: Theoretical framework and first implementation. Accid. Anal. Prev. 426 , $10 \quad 1637-1646$.

11 Li, X.R., Jilkov, V.P., 2003. Survey of Maneuvering Target Tracking. Part I: Dynamic Models. 12 IEEE Trans. Aerosp. Electron. Syst. $394,1333-1364$.

13 Mohr, D., Muller, N., Krieg, A., Gao, P., Kaas, H.-W., Krieger, A., Hensley, R., 2013. The road 14 to 2020 and beyond: What's driving the global automotive industry? McKinsey Co. 24.

15 Nadimi, N., Behbahani, H., Shahbazi, H., 2016. Calibration and validation of a new time-based 16 surrogate safety measure using fuzzy inference system. J. Traffic Transp. Eng. (English Ed. $17 \quad 31,51-58$.

18 Naito, A., Miyajima, C., Nishino, T., Kitaoka, N., Takeda, K., 2009. Driver evaluation based on 19 classification of rapid decelerating patterns. ICVES 2009 - 2009 IEEE Int. Conf. Veh.

20 Electron. Saf. $108-112$. 
1 Najafabadi, M.M., Villanustre, F., Khoshgoftaar, T.M., Seliya, N., Wald, R., Muharemagic, E.,

2 2015. Deep learning applications and challenges in big data analytics. J. Big Data 2 1, 1-

31.

4 NHTSA, 2015. Critical reasons for crashes investigated in the national motor vehicle crash

5 causation survey. (traffic safety facts crash•Stats). 13th Int. IEEE Conf. Intell. Transp. Syst.

$62-3$.

7 O’Reilly, U.-M., Yu, T., Riolo, R., Worzel, B., 2004. Genetic Programming Theory and Practice

8 II. Springer Science \& Business Media.

9 Ozbay, K., Yang, H., Bartin, B., Mudigonda, S., 2008. Derivation and Validation of a New

10 Simulation-based Surrogate Safety Measure. Transp. Res. Rec. J. Transp. Res. Board 2083,

$11 \quad 105-113$.

12 Papazikou, E., Quddus, M., Thomas, P., 2017. Detecting Deviation from Normal Driving Using

13 SHRP 2 NDS Data. Proc. 96th Annu. Meet. Transp. Res. Board August 2016, 0-15.

14 Papazikou, E., Quddus, M., Thomas, P., Kidd, D., 2019. What came before the crash? An

15 investigation through SHRP2 NDS data. Saf. Sci. 119 January 2018, 150-161.

16 Qu, Xiaobo, Kuang, Y., Oh, E., Jin, S., 2014. Safety Evaluation for Expressways: A

17 Comparative Study for Macroscopic and Microscopic Indicators. Traffic Inj. Prev. 151 ,

$18 \quad 89-93$.

19 Qu, X, Yang, Y., Liu, Z., Jin, S., Weng, J., 2014. Potential crash risks of expressway on-ramps

20 and off-ramps: a case study in Beijing, China. Saf. Sci. 70, 58-62.

21 Rodríguez, S.A., Frémont, V., Bonnifait, P., Cherfaoui, V., 2010. Visual confirmation of mobile 

objects tracked by a multi-layer lidar. IEEE Conf. Intell. Transp. Syst. Proceedings, ITSC $849-854$.

3 Songchitruksa, P., Tarko, A., 2006. Practical method for estimating frequency of right-angle 4 collisions at traffic signals. J. Transp. Res. Board 89-97.

5 Tractica, 2016. Advanced Driver Assistance System Market Forecasts.

6 Vogel, K., 2003. A comparison of headway and time to collision as safety indicators. Accid. $7 \quad$ Anal. Prev. $353,427-433$.

8 Wang, C., Quddus, M.A., Ison, S.G., 2013. The effect of traffic and road characteristics on road 9 safety: A review and future research direction. Saf. Sci. 57, 264-275.

10 Wang, S., Liu, W., Wu, J., Cao, L., Meng, Q., Kennedy, P.J., 2016a. Training Deep Neural 11 Networks on Imbalanced Data Sets. 2016 Int. Jt. Conf. Neural Networks 4368-4374.

12 Wang, S., Liu, W., Wu, J., Cao, L., Meng, Q., Kennedy, P.J., 2016b. Training deep neural 13 networks on imbalanced data sets. Proc. Int. Jt. Conf. Neural Networks 2016-Octob, 4368$14 \quad 4374$.

15 Wang, T., Wang, C., Qian, Z., 2017. Development of a new conflict-based safety metric for freeway exit ramps. Adv. Mech. Eng. 99 , 1-10.

17 Wang, X., Fan, T., Chen, M., Deng, B., Wu, B., Tremont, P., 2015. Safety modeling of urban 18 arterials in Shanghai, China. Accid. Anal. Prev. 83, 57-66.

19 Xu, C., Wang, W., Liu, P., 2013a. A genetic programming model for real-time crash prediction 20 on freeways. IEEE Trans. Intell. Transp. Syst. 142, 574-586. 
1 Xu, C., Wang, W., Liu, P., 2013b. Identifying crash-prone traffic conditions under different

2 weather on freeways. J. Safety Res. 46, 135-144.

3 Yamashita, R., Nishio, M., Do, R.K.G., Togashi, K., 2018. Convolutional neural networks: an

4 overview and application in radiology. Insights Imaging 9 4, 611-629.

5 Yang, K., Wang, X., Quddus, M.A., Yu, R., 2018. Deep learning for real-time crash prediction 6 on urban expressways using highly imbalanced big data. J. Transp. Res. Board.

7 Yang, K., Yu, R., Wang X, X., Quddus, M., 2017. Utilizing stochastic gradient descent for 8 model updating in real-time crash risk prediction. Transp. Res. Board 96th Annu. Meet.

9 Yang, M., Wang, X., Quddus, M., 2019. Examining lane change gap acceptance, duration and 10 impact using naturalistic driving data. Transp. Res. Part C Emerg. Technol. 104 April , 31711331.

12 Yu, R., Abdel-Aty, M., 2014. An optimal variable speed limits system to ameliorate traffic safety 13 doi:10.1016/j.trc.2014.05.016

14 Yu, R., Abdel-Aty, M., 2013. Utilizing support vector machine in real-time crash risk evaluation. 15 Accid. Anal. Prev. 51, 252-259.

16 Zaki, M., Sayed, T., Tageldin, A., Hussein, M., 2013. Application of Computer Vision to 17 Diagnosis of Pedestrian Safety Issues. Transp. Res. Rec. 2393 , 75-84.

18 Zhao, Z.-Q., Zheng, P., Xu, S., Wu, X., 2018. Object Detection with Deep Learning: A Review $19 \quad 148$.

20 Zheng, B., Liang, H., Zhu, Q., Yu, H., Lin, C.W., 2016. Next generation automotive architecture 

modeling and exploration for autonomous driving. Proc. IEEE Comput. Soc. Annu. Symp. VLSI, ISVLSI 53-58.

3 Zheng, L., Ismail, K., Meng, X., 2014. Traffic conflict techniques for road safety analysis: open 4 questions and some insights. Can. J. Civ. Eng. 41 7, 633-641. 